\title{
GLR test for OFDM system identification using pilot tones pattern
}

\author{
Mohamed-Rabie Oularbi ${ }^{*}$, Saeed Gazor ${ }^{2}$, Sebastien Houcke ${ }^{1}$ and Abdeldjalil Aïssa-El-Bey ${ }^{1}$
}

\begin{abstract}
In the context of cognitive network architecture, an opportunistic cognitive receiver must identify the present active networks. In this article, we propose an efficient algorithm for the identification of OFDM networks exploiting the pilot patterns used in these standards which are prescribed uniquely by their underlying standards. These pilots are inserted for the channel estimation and synchronization between the base stations and their users. The proposed generalized likelihood ratio test (GLRT) not only allows a cognitive observer to detect the active networks by analyzing the observed signals but also performs channel estimation, time-frequency synchronization as well as estimation of the noise variance. These informations are of a great interest for Quality of Service estimation in the purpose of an association with the base station. The proposed solution is applicable to the existing standards (e.g., LTE, WiMAX, WiFi), doesn't require any signaling overhead to be embedded on the pilot tones, is computationally inexpensive and only requires to know the pilot patterns. An other GLRT is proposed as a pre-detector which ignores the pilot information and allows to reduce the computational cost of the system for the cases where a large number of patterns/systems are to be tested.
\end{abstract}

\section{Introduction}

The rapid growth of wireless communications resulted in the proliferation of wireless devices and services. The demand for radio spectrum is dramatically increasing, while, most of the usable electromagnetic spectrum has already been licensed out. This policy has led to a sporadic use and shortage of the spectrum for new emerging wireless applications. To remedy this inefficiency, dynamic spectrum access techniques are proposed $[1,2]$ allowing users to share spectrum where some licensed bands are opened to unlicensed cognitive users provided that they do not produce a perceptible interference to the primary users. This culminated in the formation of the IEEE 802.22 working-group [3] which aims at using the cognitive radio (CR) technology $[4,5]$ as a key to dynamic spectrum access in the prescribed portion of TV frequency spectrum [6]. Cognition is defined as the capability of sensing the radio environment and evaluating the capabilities of alternate configurations. This enables a more flexible, efficient and reliable spectrum utilization. In such a context, the need

\footnotetext{
*Correspondence: mohamed.oularbi@telecom-bretagne.eu

${ }^{1}$ Institut Telecom, Telecom Bretagne, UMR CNRS 3192 Lab-STICC, Université

Européenne de Bretagne, Bretagne, France

Full list of author information is available at the end of the article
}

to identify the network appears when a cognitive receiver attempt to initiate a connection to a network. In fact, the cognitive users have to distinguish the IEEE 802.22 signals form the signals emitted by the TV broadcasting.

A second context where the cognitive receiver has to detect the activity of compatible surrounding wireless networks, is when applying the concept of always best connected (ABS) [7]. The concept of ABS allows multi-mode cognitive devices to move between different technologies in order to approach the QoS requirement. Seamless cognitive immigration from one technology to another one is known as vertical handover [8,9]. Before triggering a vertical handover, an opportunistic cognitive device has no knowledge about the active surrounding networks and must identify them. In a static spectrum allocation, the devices can easily detect the activities in their allocated spectrum bands using some existing technique such as energy detection [10], matched filters [11], etc. Unfortunately in cognitive networks, the allocated spectrum to the base stations (BSs) changes dynamically over time and space. Under those conditions a CR device must be enabled to identify passively the active compatible systems which are accessible in a given set of frequency bands. The spectrum is divided into a finite number of

\section{Springer}

(c) 2013 Oularbi et al: licensee Springer. This is an Open Access article distributed under the terms of the Creative Commons Attribution License (http://creativecommons.org/licenses/by/2.0), which permits unrestricted use, distribution, and reproduction in any medium, provided the original work is properly cited. 
sub-bands. The goal is to develop efficient algorithms for such a task in each sub-band. An intuitive approach is to perform detection using the cross-correlation between the observed signal and the known preamble sequence of the network of interest. Unfortunately, this approach involves long delays, for example, in the IEEE 802.22, the super-frame preamble is broad-casted only once every $160 \mathrm{~ms}$ [12]. Since, the cognitive device must sense multiple sub-bands, such a delay is not tolerable and makes this approach too expensive. In addition, this approach does not make use of extra knowledge about the structure of the signals in different networks (e.g., the involved pilot signals). In this article, we propose an alternative solution which exploits the known pilot patterns of the involved standards.

The orthogonal frequency division multiplexing (OFDM) is used in the physical layer of many of the existing networks and is also provisioned as the best candidate for most of future cognitive networks [13]. The OFDM is advantageous in cognitive networks not only because of its flexibility and scalability but more importantly because it is deemed that it allows simpler management of spectral resources.

We can categorize the existing algorithms for OFDM systems identification in four groups. The first group proposes some blind techniques [14-16] that permit to extract the OFDM signal parameters and then search those values in a lookup table to determine the corresponding system. These techniques have a high computational cost, and only the inter-carrier spacing appears to be a good signature for the actual existing systems [14]. In addition, new radio architectures such as the cognitive radio systems are converging to a common physical layer. For such future systems, it is very probable that this signature will be no longer available. The second group exploits the OFDM signal properties such as cyclic prefix (CP) cyclo-stationarity [17-19] to extract the inter-carrier spacing. Unfortunately, the performance of the CP cyclostationarity based algorithms degrades as the length of the channel impulse response approaches the cyclic prefix duration. In [20], CP and preamble cyclo-stationarity are jointly used. However, independent cognitive receivers often miss to catch the preamble, as they observe the signals at random time intervals. Specially for reducing the power consumption, the cognitive receivers shall acquire signals samples only over short intervals. In such cases, the preamble is highly likely lost, which leads to loss of the cyclo-stationary property. The third group proposes to embed some specific signatures in the physical signal and broadcast it, always. To allow unique identification of all possible co-existing systems, a number of signatures must be provisioned which adds spectral overhead. The cognitive devices must be able to regenerate these signatures and manipulate the received signals at low cost (i.e., the computational complexity must be reasonable). Authors in [21-23] proposed to dedicate some selected sub-carriers and induce a cyclo-stationary signature. Unfortunately such a technique add overhead, reduce network capacity and are not applicable for the existing OFDM networks. Finally, in [24-26], authors proposed to exploit the pilot patterns which is a signature already embedded in many existing OFDM networks (for example in WiFi, WiMAX, LTE). These pilot signals are transmitted for other reasons such as synchronization [27-29] and channel estimation [30-32]. This approach is more efficient since no spectral overhead is needed. In [25], a method is proposed for a comb-type configuration of the pilots where assuming a finite sequence of pilots. This method is only efficient for the assumed conditions. Unfortunately, the technique proposed in [26] is dedicated only to LTE signals. The first proposed method in [24] relies on the periodic redundancy often induced between pairs of pilot symbols. The second proposed method is dedicated to the case where the pilots are modulated by a pseudo random sequence, authors in [24] proposed to exploit the properties of the pseudo random generator. These methods require the knowledge of the position of the pilots in time and frequency, and are only applicable if these pilots have some known redundant relation (either in the form of known correlation or in the form of a pseudo random sequence), which make them not applicable in all cases. We must note that these pilot tones are often modulated initially by a pseudo random sequence and then by a binary phase-shift keying (BPSK) or a quadrature phase-shift keying (QPSK) signal to carry system control information; i.e., the modulating sequences are usually unknown to the third part observers. For example BPSK is used in WiFi and WiMAX and QPSK is used in LTE. Thus, we develop a method to identify the activity of such a system which is applicable under absence of the knowledge about the pilot modulating sequence.

In this article, we propose a method that exploit the pilot positions, and assume that they are modulated by a PSK signal. The proposed method is applicable to all existing standards since it does not require any knowledge or redundancy in the modulating symbols. A generalized likelihood ratio (GLR) detector which estimates the unknown channel gain, the unknown pilot modulating sequence, the noise variance and also performs time-frequency synchronization is here proposed. Thus, this method also allows third party observers to read the control channel information.

The remaining of the article is organized as follows. In Section 2, we present the OFDM signal model and formulate our detection problem. The GLR test is developed in Section 3.1. A pre-detection scheme is proposed in Section 3.2. An architecture for the receiver 
is proposed in Section 4. The Simulations results are detailed in Section 5. The synchronization impairments and the data modulating sequence impact on the performance of the algorithm are discussed in Sections 5.2 and 5.3, respectively. Finally, Section 6 concludes the article.

\section{Signal model and problem statement}

We assume that in a given frequency band, a transmitted OFDM signal consists of $N$ sub-carriers, the discrete-time baseband equivalent transmitted signal is given by

$$
x(m)=\sqrt{\frac{E_{s}}{N}} \sum_{n \in \mathbb{Z}} \sum_{k=0}^{N-1} c_{n, k} e^{2 i \pi \frac{k}{N}(m-D-n(N+D))} g(m-n(N+D)),
$$

where $E_{s}$ is the average signal power, $n$ and $k$ are the time and the frequency indexes, respectively, $c_{n, k}$ is the $n$th symbol on subcarrier $k, D$ is the cyclic prefix length, and $g(m)$ is the pulse shaping filter. The modulating symbol $c_{n, k}$ is either a data symbol $d_{n, k}$ or a pilot symbol $p_{n, k}$ as follows

$$
c_{n, k}=\left\{\begin{array}{l}
p_{n, k} \text { if } \quad(n, k) \in \mathcal{P} \\
d_{n, k} \text { otherwise }
\end{array},\right.
$$

where the pilot pattern $\mathcal{P}$ represents the set of all pilot positions $v=(n, k)$ such that $n$ and $k$ are the temporal location and the subcarrier index of a pilot, respectively. We here assume that the pilot pattern $\mathcal{P}$ is known by the receiver and that the data symbols $d_{n, k}$ are independent and identically distributed (i.i.d). In Section 5, we extend the proposed derivation to include the time and frequency synchronization. Interestingly, the pilot sequence $p_{n, k}$ is either a QPSK or BPSK signal in existing systems, i.e., $p_{n, k} \in\{ \pm 1, \pm \sqrt{-1}\}$. In this article, we exploit this property in order to detect if a given pattern $\mathcal{P}$ with such a modulation is present.

As an example, Figure 1 shows the configuration of the IEEE 802.16e downlink-partial usage of sub-channels (DL-PUSC). There is $N=512$ (the FFT length) subcarriers which include 360 data-sub-carriers, 60 pilotsubcarriers and 92 null-subcarriers (46 left guard band and 45 right guard band and one DC). The pilot signals are dedicated for various estimation purposes and are reproducible at the receiver. No signal is transmitted in the null-subcarriers in order to enable the signal to naturally decay and create the FFT "brick wall" shaping [33]. In this example, the set $\mathcal{P}$ is defined as

$$
\mathcal{P}=\left\{\left(n, k\left(n, n_{c}, q\right)\right) \mid n_{c}=0, \ldots, 29, n \in \mathbb{Z} q \in\{0,1\}\right\},
$$

where

$$
k\left(n, n_{c}, q\right)= \begin{cases}50+14 n_{c}+4 q, & n_{c}<15 \text { and } n \text { is even, } \\ 50+14 n_{c}+4 q+1, & n_{c} \geq 15 \text { and } n \text { is even, } \\ 46+14 n_{c}+4 q, & n_{c}<15 \text { and } n \text { is odd } \\ 46+14 n_{c}+4 q+1, & \text { otherwise. }\end{cases}
$$

We must note that our proposed algorithm is applicable to other standards where the pilot pattern is defined differently.

Let $\{h(l)\}_{l=0}^{L-1}$ denotes the baseband equivalent discretetime finite impulse response of the channel. In the derivation of our algorithm, we assume that the channel is time invariant and $L<D$. However, our simulation results reveal that the proposed algorithm performs well in slow time varying channels (see example in Section 5). The received samples of the OFDM signal are expressed as

$$
y(m)=e^{-j\left(2 \pi \varepsilon \frac{m-\tau}{N}+\varphi\right)} \sum_{l=0}^{L-1} h(l) x(m-l-\tau)+w(m),
$$

where we assume that the additive noise $w(m)$ is a circularly symmetrical complex white Gaussian (CSCWG) process, i.e., $w(m) \sim \mathcal{N}\left(0, \sigma^{2}\right)$ with zero mean and unknown variance of $\sigma^{2}$. We define $\varepsilon$ as the carrier frequency offset (normalized by the sub-carrier spacing) between the active system and the observer, $\varphi$ is the initial arbitrary phase mismatch, $\tau$ is the timing delay. For convenience and easy tailoring of our algorithm, we first assume that the system is synchronized, i.e., $(\varepsilon, \tau, \varphi)$ is known and is pre-compensated to be zero. This can be realized using any existing algorithm in the literature. In Section 5, we show how the proposed algorithm can be used to perform the synchronization, or to initialize any other synchronization algorithm. Applying a fast Fourier

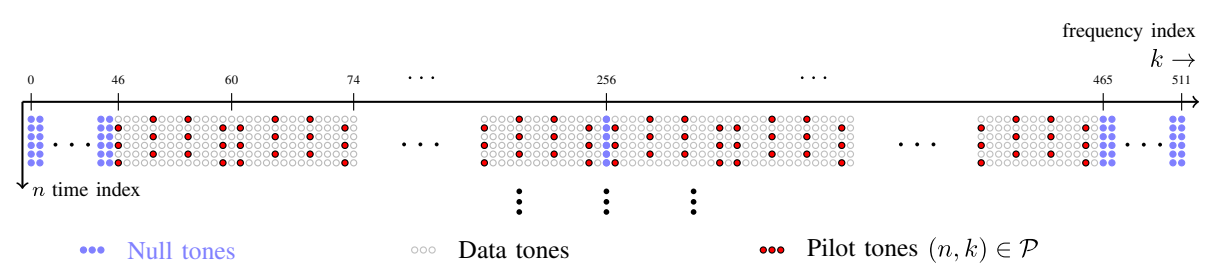

Figure 1 An example for pilot pattern configuration (for 802.16e WiMax DL-PUSC), $N=512$ subcarriers which include 360 data-subcarriers, 60 pilot-subcarriers and 92 null-subcarriers (46 left guard band and 45 right guard band and one DC) [33]. 
transform (FFT) to (5), it can be easily shown that the symbols $c_{n, k}$ are observed via $Y_{n, k}$ as follows (for when $(\varepsilon, \tau$, varphi $)=(0,0,0))$

$$
\begin{aligned}
Y_{n, k} & \triangleq \frac{1}{\sqrt{N}} \sum_{m=0}^{N-1} y[n(N+D)+D+m] e^{-2 i \pi \frac{k m}{N}} \\
& =H_{k} c_{n, k}+W_{n, k},
\end{aligned}
$$

where $\mathbf{H}=\left[H_{0}, H_{1}, \ldots, H_{N}\right]=\sqrt{\frac{E_{s}}{N}} \operatorname{FFT}[h(0), h(1), \ldots$, $h(L-1), 0, \ldots, 0]$ is the channel frequency response. Since the applied transformation in (6) is orthonormal, the noise process $W_{n, k}$ in (7) is also a CSCWG process with zero mean and unknown variance of $\sigma^{2}$. We can rewrite (7) in a matrix form as

$$
\mathbf{Y} \triangleq\left[\begin{array}{cccc}
Y_{1,0} & Y_{1,1} & \cdots & Y_{1, N-1} \\
\vdots & & \ddots & \vdots \\
Y_{M, 0} & Y_{M, 1} & \cdots & Y_{M, N-1}
\end{array}\right]
$$

Let $\mathcal{H}_{0}$ and $\mathcal{H}_{1}$ denote the hypotheses that the OFDM signal is absent or present, respectively. Under $\mathcal{H}_{0}$ the first term in (7) is absent and the observed signals has an i.i.d Gaussian distribution, i.e.,

$$
f\left(\mathbf{Y} ; \mathcal{H}_{0}, \sigma^{2}\right)=\frac{1}{\left|\pi \sigma^{2}\right|^{M N}} \exp \left(-\frac{\|\mathbf{Y}\|^{2}}{\sigma^{2}}\right),
$$

where $\|\mathbf{Y}\|^{2}=\operatorname{trace}\left(\mathbf{Y} \mathbf{Y}^{H}\right)$, and ()$^{H}$ stands for the Hermitian transpose.

Under $\mathcal{H}_{1}$ two scenarios are possible. Either an OFDM system is active with the desired pilot pattern $\mathcal{P}$ or $\mathbf{Y}$ is produced by another OFDM network with an unknown pilot pattern. In this section, we propose a GLR detector to test $\mathcal{H}_{1}$ for a known pilot pattern. In Section 3.2, we recommend an algorithm as a pre-detection process which detects the activity of a system without using the pilot pattern information. This inexpensive detector allows us to reduce the computational cost without any performance loss. In this section, we test $\mathcal{H}_{0}$ against $\mathcal{H}_{1}$ given a known pattern $\mathcal{P}$.

Under $\mathcal{H}_{1}$, we now assume that the signal $c_{n, k}$ is present including a given pilot pattern $\mathcal{P}$ as in (2). We use $v=$ $(n, k)$ as the time-frequency pair index. We denote $\mathcal{P}_{k}=$ $\{(n, k) \mid \forall n\} \cap \mathcal{P}$ as the subset of pilot indexes for a given sub-carrier $k$. It is obvious that $\left\{\mathcal{P}_{k}\right\}_{k=0}^{N-1}$ is a partition for $\mathcal{P}$. We denote $\overline{\mathcal{P}}_{k}=\{(n, k) \mid \forall n\}-\mathcal{P}$ as the subset of time-frequency pair indexes which are not pilot for a given frequency index $k$. The cardinal number of a set $\mathcal{A}$ is denoted by $|\mathcal{A}|$. Since the transmitter uses the adaptive modulation and coding (AMC), and disposing of no information about the distribution of the received data symbols, we assume that the samples situated on a given sub-carrier $k$ follows a complex Gaussian distribution of zeros mean and a variance $\sigma_{k}^{2}$, constant over a given $k$.
Under provided that $v \in \mathcal{P}$ we have $\left(Y_{v}-H_{k} c_{v} ; v \in \mathcal{P}\right) \sim$ $\mathcal{N}\left(0, \sigma^{2}\right)$, and in such a case we get

$$
\begin{aligned}
& f\left(\mathbf{Y} ; \mathcal{H}_{1}, \sigma^{2}, \mathbf{C}, \mathbf{H}, \sigma_{k}^{2}\right) \\
& =\prod_{k}\left(\prod_{\nu \in \mathcal{P}_{k}} \frac{1}{\pi \sigma^{2}} e^{-\frac{1}{\sigma^{2}}\left|Y_{\nu}-H_{k} c_{\nu}\right|^{2}} \prod_{\nu \in \overline{\mathcal{P}}_{k}} \frac{1}{\pi \sigma_{k}^{2}} e^{-\frac{1}{\sigma_{k}^{2}}\left|Y_{\nu}\right|^{2}}\right),
\end{aligned}
$$

where $\mathbf{C}$ is defined as the vector of transmitted pilot symbols with a length of $|\mathcal{P}|$.

\section{Proposed algorithms}

In this section, we first introduce the identification algorithm. Then by ignoring the pilot pattern we propose a pre-detection algorithm that allows to considerably reduce the computational cost of the identification process.

\subsection{Identification algorithm}

Given Equations (9) and (10), the problem of detecting an OFDM signal using a pilot pattern $\mathcal{P}$ can be expressed by the following binary hypothesis test:

$$
\left\{\begin{aligned}
& f\left(\mathbf{Y} ; \mathcal{H}_{0}, \sigma^{2}\right)=\frac{1}{\left(\pi \sigma^{2}\right)^{M N}} \exp \left(-\frac{1}{\sigma^{2}}\|\mathbf{Y}\|^{2}\right) \\
& f\left(\mathbf{Y} ; \mathcal{H}_{1}, \sigma^{2}, \mathbf{C}, \mathbf{H}, \sigma_{k}^{2}\right)=\prod_{k}\left(\prod_{v \in \mathcal{P}_{k}} \frac{1}{\pi \sigma^{2}} e^{-\frac{1}{\sigma^{2}}\left|Y_{v}-H_{k} c_{v}\right|^{2}}\right. \\
&\left.\times \prod_{v \in \overline{\mathcal{P}}_{k}} \frac{1}{\pi \sigma_{k}^{2}} e^{-\frac{1}{\sigma_{k}^{2}}\left|Y_{v}\right|^{2}}\right) .
\end{aligned}\right.
$$

To derive the GLR detector we first maximize (9) with respect to $\sigma^{2}$ and (10) with respect to $\left(\sigma^{2},\left\{\sigma_{k}^{2}\right\}\right)$ and obtain the ML estimates of $\sigma^{2}$ and $\sigma_{k}^{2}$, respectively, as follows

$$
\begin{aligned}
& \mathcal{H}_{0}: \widehat{\sigma^{2}}=\frac{1}{M N}\|\mathbf{Y}\|^{2} \\
& \mathcal{H}_{1}:\left\{\begin{array}{l}
\widehat{\sigma_{k}^{2}}=\frac{\sum_{v \in \overline{\mathcal{P}_{k}}}\left|Y_{v}\right|^{2}}{\left|\overline{\mathcal{P}_{k}}\right|} \\
\widehat{\sigma^{2}}=\frac{\sum_{k} \sum_{v \in \mathcal{P}_{k}}\left|Y_{v}-H_{k} c_{v}\right|^{2}}{|\mathcal{P}|} .
\end{array}\right.
\end{aligned}
$$

Substituting (12) and (13), respectively, in the PDFs in (11), we find

$$
f\left(\mathbf{Y} ; \mathcal{H}_{0}, \widehat{\sigma^{2}}\right)=\frac{e^{-M N}}{\left(\frac{\pi}{M N}\|\mathbf{Y}\|^{2}\right)^{M N}}
$$




$$
\begin{aligned}
f\left(\mathbf{Y} ; \mathcal{H}_{1}, \widehat{\sigma^{2}}, \widehat{\sigma_{k}^{2}}\right) & =\frac{e^{-M N}}{\left(\frac{\pi}{|\mathcal{P}|} \sum_{k} \sum_{v \in \mathcal{P}_{k}}\left|Y_{v}-H_{k} c_{v}\right|^{2}\right)^{|\mathcal{P P}|}} \\
& \times \prod_{k}\left(\frac{\pi}{\left|\overline{\mathcal{P}_{k}}\right|} \sum_{v \in \overline{\mathcal{P}}_{k}}\left|Y_{v}\right|^{2}\right)^{-\left|\overline{\mathcal{P}_{k}}\right|}
\end{aligned}
$$

Now, we have to maximize (15) with respect to $\mathbf{C}$ and $\mathbf{H}$. This is equivalent to minimize $\sum_{v \in \mathcal{P}_{k}}\left|Y_{\nu}-H_{k} c_{\nu}\right|^{2}$ with respect to $H_{k}$ and the pilot symbols for all sub-carriers $k$ with $\mathcal{P}_{k} \neq \emptyset$. The elements $c_{v}$ are PSK symbols. For this case, a fast optimal algorithm recently proposed in [34] that can be employed for this optimization problem with a computational complexity order of $\left|\mathcal{P}_{k}\right| \log _{2}\left(\left|\mathcal{P}_{k}\right|\right)$ per sub-carrier. Using this fast algorithm in [34] the computational cost of this optimization is $\sum_{k}\left|\mathcal{P}_{k}\right| \log _{2}\left(\left|\mathcal{P}_{k}\right|\right)$. A suboptimal algorithm is also proposed in [35] (involves very small performance loss) with a reduced computational complexity order of $\left|\mathcal{P}_{k}\right|$ per sub-carrier and hence its overall computational cost is $|\mathcal{P}|$.

We provide the data $\left\{Y_{v}\right\}_{v \in \mathcal{P}_{k}}$ to the procedure in [34] and obtain the ML estimates of the symbols $c_{\nu}$ hereafter denoted by $\widehat{c_{\nu}}$. In this case, the ML estimate of $H_{k}$ is given by

$$
\widehat{H_{k}}=\frac{1}{\left|\mathcal{P}_{k}\right|} \sum_{\nu \in \mathcal{P}_{k}}{\widehat{c_{\nu}}}^{H_{Y_{\nu}}}
$$

Substituting (16) and $\widehat{c_{v}}$ obtained from the procedure in [34] in (??), the ML estimate of $\sigma^{2}$ under $\mathcal{H}_{1}$ becomes

$$
\widehat{\sigma^{2}}=\frac{1}{|\mathcal{P}|} \sum_{k}\left(\sum_{v \in \mathcal{P}_{k}}\left|Y_{v}\right|^{2}-\frac{1}{\left|\mathcal{P}_{k}\right|}\left|\sum_{\nu \in \mathcal{P}_{k}}{\widehat{c_{v}}}^{H} Y_{\nu}\right|^{2}\right)
$$

Substituting (17) in (11) under $\mathcal{H}_{0}$, the ratio of the likelihood functions becomes
Equivalently, the Log-Likelihood Ratio $\mathcal{L}(\mathbf{Y}, \mathcal{P})=$ $\log (L(\mathbf{Y}, \mathcal{P}))$ is

$$
\begin{aligned}
\mathcal{L}(\mathbf{Y}, \mathcal{P}) & =-M N \log (M N)+M N \log \left(\|\mathbf{Y}\|^{2}\right)+|\mathcal{P}| \log (|\mathcal{P}|) \\
& -|\mathcal{P}| \log \left(\sum_{k}\left(\sum_{\nu \in \mathcal{P}_{k}}\left|Y_{\nu}\right|^{2}-\frac{1}{\left|\mathcal{P}_{k}\right|}\left|\sum_{\nu \in \mathcal{P}_{k}}{\widehat{\mathcal{C}_{v}}}^{H} Y_{v}\right|^{2}\right)\right) \\
& +\sum_{k}\left|\overline{\mathcal{P}_{k}}\right| \log \left(\left|\overline{\mathcal{P}_{k}}\right|\right)-\sum_{k}\left|\overline{\mathcal{P}_{k}}\right| \log \left(\sum_{\nu \in \overline{\mathcal{P}_{k}}}\left|Y_{\nu}\right|^{2}\right) .
\end{aligned}
$$

In the above, the terms $M N \log (M N),|\mathcal{P}| \log (|\mathcal{P}|)$ and $\sum_{k}\left|\overline{\mathcal{P}_{k}}\right| \log \left(\left|\overline{\mathcal{P}_{k}}\right|\right)$ are constants. Thus, we define the following statistic test to decide if the pattern is present or not

$$
\begin{aligned}
\mathcal{T}(\mathbf{Y}, \mathcal{P})= & M N \log \left(\|\mathbf{Y}\|^{2}\right) \\
& -|\mathcal{P}| \log \left(\sum_{k}\left(\sum_{v \in \mathcal{P}_{k}}\left|Y_{v}\right|^{2}-\frac{1}{\left|\mathcal{P}_{k}\right|}\left|\sum_{\nu \in \mathcal{P}_{k}}{\widehat{c_{v}}}^{H} Y_{\nu}\right|^{2}\right)\right) \\
& -\sum_{k}\left|\overline{\mathcal{P}_{k}}\right| \log \left(\sum_{v \in \overline{\mathcal{P}_{k}}}\left|Y_{v}\right|^{2}\right) \underset{\mathcal{H}_{0}}{\stackrel{\mathcal{H}_{1}}{\gtrless}} \eta_{\text {det }},
\end{aligned}
$$

where the detection threshold $\eta_{\operatorname{det}}$ can be obtained by Monte-carlo simulation, assuming that the pattern $\mathcal{P}$ is absent (see Section 5 for more details). This detection threshold is independent of the SNR and of the noise variance. Indeed, one can check easily that the test statistic presented in (20) is invariant to the transformation $G(\mathbf{Y})=\alpha \mathbf{Y}$, where $\alpha$ is an arbitrary number. This makes it computable as summarized in Algorithm 1 without the knowledge of these two lasts parameters.

The proposed algorithm performs an $N$-point Fast Fourier Transform (FFT) on $M$ frames, and then detects $|\mathcal{P}|$ PSK symbol by the algorithm proposed in [34]. Taking into account the complexity of each term in (20), the overall Computational Complexity (CC) is $M N\left(2+\log _{2} N\right)+$ $\sum_{k}\left|\mathcal{P}_{k}\right| \log _{2}\left|\mathcal{P}_{k}\right|+|\mathcal{P}|$ for $M$ frames using the optimal algorithm in [34] and $M N\left(2+\log _{2} N\right)+2|\mathcal{P}|$ using the suboptimal algorithm in [35]. For example in [24], authors have considered a case with $M=24,30$ pilots pairs

$$
\begin{aligned}
L(\mathbf{Y}, \mathcal{P}) & =\frac{\sup _{\sigma^{2}, \mathbf{C}, \mathbf{H}, \sigma_{k}^{2}} f\left(\mathbf{Y} ; \mathcal{H}_{1}, \sigma^{2}, \mathbf{C}, \mathbf{H}, \sigma_{k}^{2}\right)}{\sup _{\sigma^{2}} f\left(\mathbf{Y} ; \mathcal{H}_{0}, \sigma^{2}\right)} \\
& =\frac{\left(\frac{1}{M N}\|\mathbf{Y}\|^{2}\right)^{M N}}{\left(\frac{1}{|\mathcal{P}|} \sum_{k}\left(\sum_{\nu \in \mathcal{P}_{k}}\left|Y_{\nu}\right|^{2}-\frac{1}{\left|\mathcal{P}_{k}\right|}\left|\sum_{\nu \in \mathcal{P}_{k}}{\widehat{c_{\nu}}}^{H} Y_{\nu}\right|^{2}\right)\right)^{|\mathcal{P}|} \prod_{k}\left(\frac{1}{\left|\overline{\mathcal{P}_{k}}\right|} \sum_{\nu \in \overline{\mathcal{P}_{k}}}\left|Y_{\nu}\right|^{2}\right)^{\left|\overline{\mathcal{P}_{k}}\right|}} .
\end{aligned}
$$




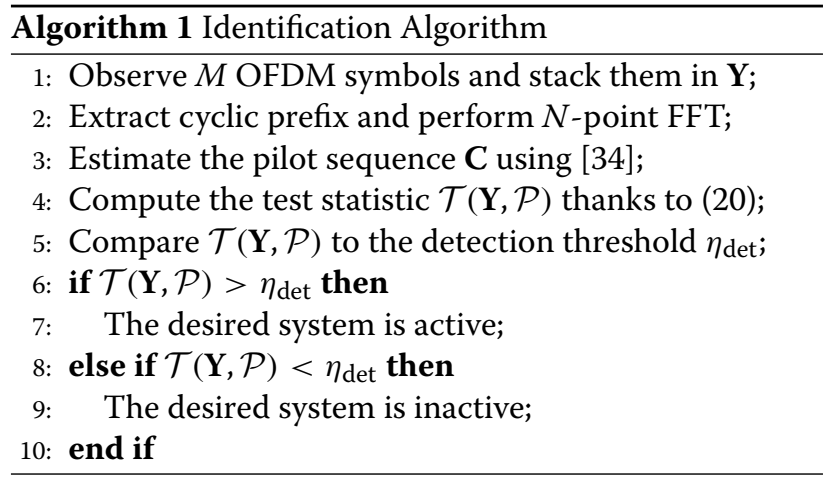

and $N=2048$. In this case, the overall complexity of their algorithm is approximately $5.4 \times 10^{5} \mathrm{MAC}$ where each MAC consists of one complex multiplication and one addition. For this case, the complexity of our proposed algorithm is $6.45 \times 10^{5}$ operations which is slightly more expensive (less than 19\%) than the one proposed in [24]. Since the computational cost of the FFT is predominant in the both algorithm, these algorithms have similar order of computational costs.

\subsection{Pre-detection algorithm}

Assume that there is multiple candidates for the pilot pattern. For such a case if none of systems are active, executing the detector proposed in the previous section is a waste of resources, specially if the pilot pattern at the receiver is not synchronized with the true value. For such a cases, we propose a coarse pre-detector to test of any system is active or not without using the information about the pilot locations. This computationally inexpensive predetection algorithm considers no information about pilot samples assuming that the corresponding symbols are Gaussian like data and is insensitive to time and frequency synchronization. We must emphasize the aim of the proposed pre-detector is not to detect PUs like in [36-39], but is to filter out the unlikely cases and to reduce the computational cost of the detection procedure, specially in the cases cited above. In this context, the detection of a system at very low SNRs is a waste of time and resources. Indeed, detecting a system in a range of SNR where our identifier does not perform well is inconvenient. In addition, a vertical handover process is only performed if a system with a reasonably high SNR is detected to satisfy the link quality condition.

Let the hypothesis $\mathcal{H}_{2}$ denotes that a system is active. Note that $\mathcal{H}_{2}$ includes $\mathcal{H}_{1}$. In this case the p.d.f of $\mathbf{Y}$ is the same as expressed in equation (10) with the exception that we must ignore $\mathcal{P}$. Therefore, the first product term is the same as the second term, i.e.,

$$
f\left(\mathbf{Y} ; \mathcal{H}_{2}, \sigma_{k}^{2}\right)=\prod_{k=0}^{N-1} \frac{1}{\left(\pi \sigma_{k}^{2}\right)^{M}} \exp \left(-\frac{1}{\sigma_{k}^{2}}\|\mathbf{Y}(k)\|^{2}\right),
$$

where $\mathbf{Y}(k)$ denotes the $k$ th column of the matrix $\mathbf{Y}$ and is assumed to have a Gaussian distribution with zero-mean and variance of $\sigma_{k}^{2}$. The ML estimate of $\sigma_{k}^{2}$ is $\widehat{\sigma_{k}^{2}}=\frac{1}{M}\|\mathbf{Y}(k)\|^{2}$ and $f\left(\mathbf{Y} ; \mathcal{H}_{2}, \widehat{\sigma_{k}^{2}}\right)=$ $\left(\frac{M}{\pi e}\right)^{M N} \prod_{k=0}^{N-1}\left(\|\mathbf{Y}(k)\|^{2}\right)^{-M}$. Thus using Equation (15), the LR function is given by

$$
\begin{aligned}
L(\mathbf{Y})= & \frac{\sup _{k}^{2} f\left(\mathbf{Y} ; \mathcal{H}_{2}, \sigma_{k}^{2}\right)}{\sup _{\sigma^{2}} f\left(\mathbf{Y} ; \mathcal{H}_{0}, \sigma^{2}\right)} \\
= & \frac{\left(\|\mathbf{Y}\|^{2}\right)^{M N}}{\prod_{k=0}^{N-1}\left(\|\mathbf{Y}(k)\|^{2}\right)^{M}} .
\end{aligned}
$$

We can easily see that $N \sqrt[M]{L(\mathbf{Y})}$ is the harmonic mean of $\left\{\frac{\|\mathbf{Y}(k)\|^{2}}{\|\mathbf{Y}\|^{2}}\right\}_{k=0}^{N-1}$. Thus, we can view the LR as a kind of average of the ratios of energies in sub-carriers to the total energy. In addition $\frac{1}{\sqrt[M N]{L(\mathbf{Y})}}$ is a measure of the spectral flatness of the data, i.e; the ratio of the geometric mean to the arithmetic mean of the sub-carriers energies. Obviously under $\mathcal{H}_{0}$ for the white Gaussian noise, the spectrum is completely flat and it is expected that $L(\mathbf{Y})$ is minimized. Otherwise, as the BS is using the AMC, the energy in different sub-carriers are not necessarily equal and hence $L(\mathbf{Y})$ is increased. Taking logarithm of (22), the log-LR for the pre-detector is defined as the decision statistic as follows

$$
\mathcal{L}(\mathbf{Y})=M N \log \left(\|\mathbf{Y}\|^{2}\right)-M \sum_{k} \log \left(\|\mathbf{Y}(k)\|^{2}\right) \underset{\mathcal{H}_{0}}{\stackrel{\mathcal{H}_{2}}{\gtrless}} \eta_{\text {pre }}
$$

which is a measure of the homogeneity index of $\left.\left\{\|\mathbf{Y}(k)\|^{2}\right)\right\}_{k=0}^{N-1}$. This pre-detector is summarized in Algorithm 2. The distribution of the ratio of the geometric mean to the arithmetic mean of random samples from a $\Gamma$ distribution has been studied in [40]. Unfortunately, the obtained expression is very hard to manipulate. In current standards, the product $M N$ is a large number ${ }^{\mathrm{a}}$. To the best of our knowledge for this detector, the exact analysis is very complicated and the only existing analysis is recently carried out by S Akhavan-Astaneh and S Gazor (unpublished work) for the asymptotic case where $M N$ is large enough. We can prove that the distribution of the decision statistic $\mathcal{L}(\mathbf{Y})$ in (24) under both $\mathcal{H}_{0}$ and $\mathcal{H}_{1}$ converges to a normal distribution as $M N \rightarrow \infty$ with means and variances that cost in our case

$$
\left\{\begin{array}{c}
E\left[\mathcal{L} ; \mathcal{H}_{0}\right]=M N(\psi(M N)-\psi(M)) \\
\operatorname{var}\left[\mathcal{L} ; \mathcal{H}_{0}\right]=M^{2}\left(N \psi^{\prime}(M)-N^{2} \psi^{\prime}(M N)\right),
\end{array}\right.
$$




$$
\left\{\begin{aligned}
E\left[\mathcal{L} ; \mathcal{H}_{1}\right]= & E\left[\mathcal{L} ; \mathcal{H}_{0}\right]+M N \Omega\left(\sigma_{0}^{2}, \ldots, \sigma_{N-1}^{2}\right) \\
\operatorname{var}\left[\mathcal{L} ; \mathcal{H}_{1}\right]= & M^{2} N\left(\psi^{\prime}(M)-N^{2} \psi^{\prime}(M N)\right) \\
& +M N\left(\frac{\sum_{k} \sigma_{k}^{4}}{\left(\sum_{k} \sigma_{k}\right)^{2}}-\frac{1}{N}\right)
\end{aligned}\right.
$$

where $\psi(z)=\Gamma^{\prime}(z) / \Gamma(z)$ is the digamma function and hereafter, we referee to $\Omega\left(\sigma_{0}^{2}, \ldots, \sigma_{N-1}^{2}\right)=$ $\log \left(\frac{\sigma_{0}^{2}+\cdots+\sigma_{N-1}^{2}}{N}\right)-\frac{\log \sigma_{0}^{2}+\cdots+\log \sigma_{N-1}^{2}}{N}$ as the homogeneity index of the bands energies $\left(\sigma_{0}^{2}, \ldots, \sigma_{N-1}^{2}\right)$. This is why a better performance is achieved when the center of the two normal pdfs are far apart, which happens only if the $\sigma_{k}^{2} \mathrm{~s}$ are heterogeneous, i.e., the source spectrum observed is non flat. Figure 2 depicts the empirical pdfs, the normal pdfs in (25) and (26) of $\mathcal{L}$ under $\mathcal{H}_{0}$ and $\mathcal{H}_{1}$, where $M=12, N=512$ and the $\sigma_{k}^{2}$ are random variables chosen uniformly in the interval $[1,2]$. This reveals that our theoretical expressions are accurate.Therefore, the probabilities of false alarm and mis-detection of $\mathcal{L}$ are approximated as follows [41]

$$
\left\{\begin{array}{l}
P_{\mathrm{fa}, \mathcal{L}} \approx Q\left(\frac{\eta_{\mathrm{pre}}-M N(\psi(M N)-\psi(M))}{\sqrt{M^{2}\left(N \psi^{\prime}(M)-N^{2} \psi^{\prime}(M N)\right)}}\right) \\
P_{\mathrm{md}, \mathcal{L}} \approx 1-Q\left(\frac{\eta_{\mathrm{pre}}-M N(\psi(M N)-\psi(M))-M N \Omega\left(\sigma_{0}^{2}, \ldots, \sigma_{N-1}^{2}\right)}{\sqrt{M^{2} N\left(\psi^{\prime}(M)-N \psi^{\prime}(M N)\right)+M N^{2}\left(\frac{\sum_{k} \sigma_{k}^{4}}{\left(\sum_{k} \sigma_{k}\right)^{2}}-\frac{1}{N}\right)}}\right),
\end{array}\right.
$$

where $Q(z)=\frac{1}{\sqrt{2 \pi}} \int_{z}^{\infty} e^{-t^{2}} d t$.

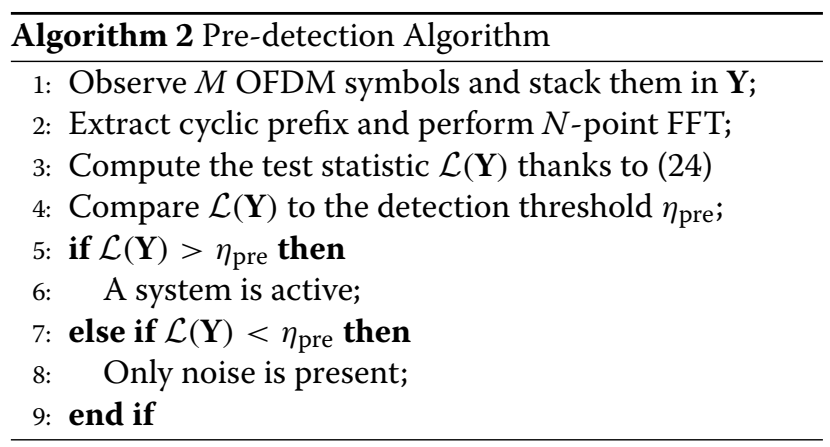

If $\mathcal{L}$ is greater than the threshold, the hypothesis $\mathcal{H}_{2}$ is validated. Only in this case, we need to test $\mathcal{H}_{1}$ against $\mathcal{H}_{0}$ using $\mathcal{P}$ and (20). We propose to chose $\eta_{\text {pre in }}$ (24) and $\eta_{\text {det }}$ in (20) such that the constant false alarm probability $P_{\text {fa,pre }}$ of (24) is slightly higher than $P_{\mathrm{fa}}$ of (20). The reason is that possible false alarms of (24) are further processed in (20). In contrast, if (24) fails to detect the activity of the system no further processing is applied. The proposed predetector has a computational cost of $M N \log N+2 M N$. Assuming that there are $N_{p}$ feasible pilot pasterns that needed to be tested, the overall computational cost of the

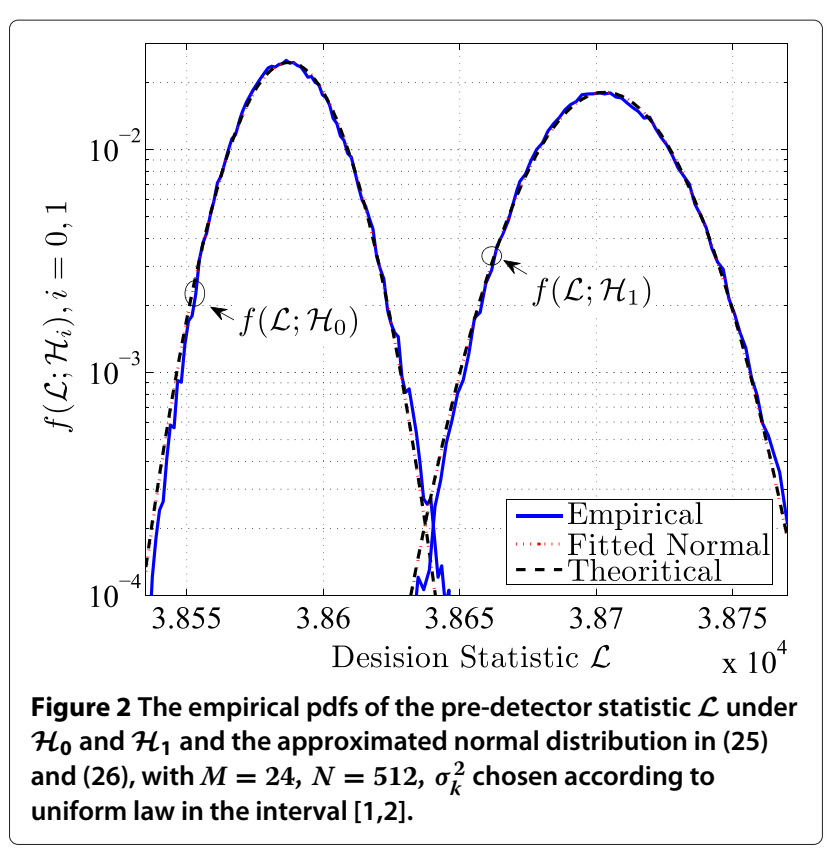

combined pre-detection and $N_{p}$ detection is $M N \log N+$ $2 M N+N_{p} P_{\text {fa,pre }}\left(\sum_{k}\left|\mathcal{P}_{k}\right| \log _{2}\left|\mathcal{P}_{k}\right|+|\mathcal{P}|\right)$ MAC. Such a combination is more cost effective for larger values of $N_{p}$ since we can set $P_{\text {fa,pre }}$ to a relatively feasible small number.

\section{Summary of the proposed sensing unit}

Our proposed detector should be implemented in the base-band processing unit $[42,43]$ of a cognitive receiver. We assume that the spectrum is divided to a finite number of frequency channels which are used by different networks. The proposed detector is used to identify and to detect the activity of these networks.

In our method, we need to assume that the sampling frequencies (and the bandwidth) used by these networks are all multiple integer factor of a constant value. This allows us to use one single module to identify all possible networks sharing the same bandwidth. We assume that $N, D$ and $\mathcal{P}$ are given for all specific networks of interest. A flow chart for the proposed spectrum sensing unit is illustrated in Figure 3. First, the sampled data are processed using FFT in order to obtain $Y_{n, k}$ as in (6). Then, the pre-detection is performed by computing LLR $\mathcal{L}(\mathbf{Y})$ and comparing it with a (pre-calculated) threshold $\eta_{\text {pre }}$ as in (24). This threshold $\eta_{\text {pre }}$ is set to satisfy a desired $P_{\mathrm{fa} \text {,pre. If }}$ this LLR $\mathcal{L}(\mathbf{Y})$ is less than the threshold, this sub-channel is declared as vacant and the next sub-channel will be processed. Otherwise if $\mathcal{L}(\mathbf{Y})>\eta_{\text {pre }}$, the system will make more investigation about all possible candidate networks, by using $\mathcal{P}$ and the test statistic expressed in (20) by comparing $\mathcal{T}(\mathbf{Y}, \mathcal{P})$ to a threshold $\eta_{\text {det }}$. The decision of (20) indicates if a network using pilot $\mathcal{P}$ is active or not. To 


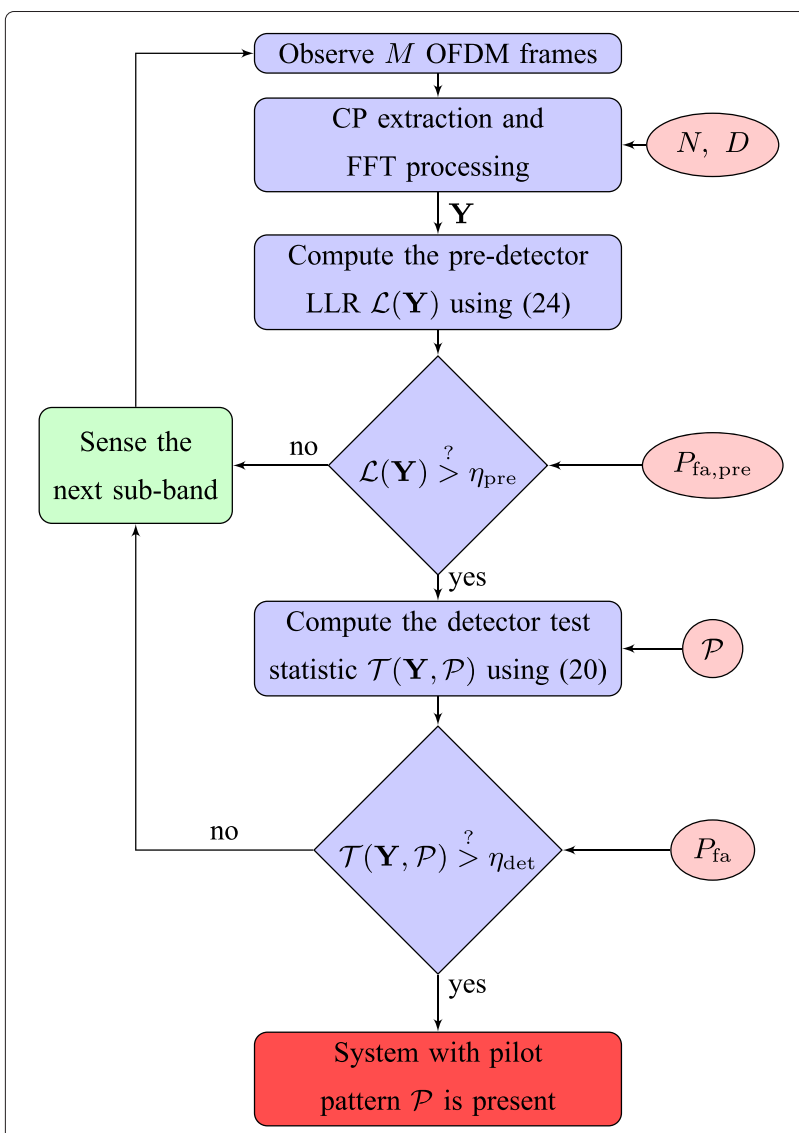

Figure 3 A flow chart for the proposed architecture for the base-band spectrum sensing unit of the cognitive receiver.

ensure that the pre-detector is not eliminating potential events that are susceptible of being active systems, its probability of false alarm must be chosen large enough. This is because it filters some of the events and the missdetection probability of a cascade system is less than that of each sub-system. Simulations in Section 5 show that to achieve good results we should choose $P_{\mathrm{fa}, \mathrm{pre}}>10 P_{\mathrm{fa}}$.

\section{Simulations}

\subsection{Synchronous case}

Simulations are investigated on OFDMA signals, all the results are averaged over 1000 Monte-Carlo runs assume a perfect synchronization. In our simulations, the thresholds are determined experimentally, as follows: the decision statistics for $10^{4}$ independent trials in the absence of signal are sorted in a descending order and the threshold is chosen as the $\% 100 \times P_{\mathrm{fa}}$-percentile of the resulting data. For example for $P_{\mathrm{fa}}=0.02$, the threshold is chosen as the $0.02 \times 10^{4}=200$ th ordered data; i.e., such that $\% 100 \times P_{\mathrm{fa}}$ of the decision statistics are above the threshold. We have chosen $M=24$ as the number of observed symbols for our simulations, (note that in WiMAX Mobile 24 symbols represents $2.5 \mathrm{~ms}$ ).
First, we analyze the performance of the pre-detector for a 512 sub-carrier OFDM system with $D=64,60$ pilots, 360 data, 91 guard and 1 DC sub-carrier. The data symbols $d_{n, k}$ are randomly chosen from a QPSK, 16-QAM or 64-QAM constellations with equal probabilities. The signal to noise ratio is defined as SNR $=10 \log _{10}\left(\frac{E_{s}}{\sigma^{2}}\right)$. The propagation channel is simulated as a discrete time frequency selective channel assuming that $\{h(l)\}_{l=0}^{L-1}$ are independent with zero-mean Gaussian distribution. We assume an exponential decay profile for the variance of $h(l)$, i.e., $E\left[|h(l)|^{2}\right]=G e^{-l / \mu}$ for $l=0, \ldots, L-1$, where $G$ is chosen such that $\sum_{l=0}^{L-1} E\left[|h(l)|^{2}\right]=1$. Figure 4 shows the probability of missed detection $\left(P_{\text {mis }}=1-P_{\mathrm{d}}\right.$, where $P_{\mathrm{d}}$ is the detection probability) versus the SNR for various values of false alarm probability. As expected, we could achieve a lower probability of missed detection without using the pilot pattern information provided that we allow a higher false alarm rate $P_{\mathrm{fa}}$ pre for the pre-detector than $P_{\mathrm{fa}}$.

Following the simulation set up in [24], we compare our proposed detector with their method. The pilot configuration is comb-type (i.e., a given sub-carrier is dedicated to pilots $\forall n$ ), the correlation induced between pilots is such that $p_{n, k}=p_{n+2, k+210}$, the pilots are BPSK symbols. We also normalize the data vector in each sub-carrier by its own standard deviation in order to obtain a less sensitive criterion to the unknown received signal gain as proposed in [24]. Figure 5 shows the performance of the proposed GLRT when compared to the pilot induced cyclo-stationarity detector (PICD) proposed in [24] for $P_{\mathrm{fa}}=0.02$ and $P_{\mathrm{fa}}=0.001$. We clearly see that the GLRT outperforms the PICD by almost a $1 \mathrm{~dB}$ gain.

As a more realistic scenario, we consider the case of Mobile WiMAX DL-PUSC configuration [33] as illustrated in Figure 1, where the pilots are BPSK symbols $2.54 \mathrm{~dB}$ boosted compared with data sub-carriers ${ }^{\mathrm{b}}$. We assume that the transmit power for all data sub-carriers are equal, and $d_{n, k}$ are randomly and independently chosen from a QPSK, 16-QAM or 64-QAM constellation. The propagation channel is generated by the same manner as in the previous simulation. We assume that $p_{n, k}=$ $p_{n, k+210}$ since the algorithm in [24] requires such a intentionally embedded correlation. Figure 6 compares the performance of GLRT with that of the PICD for Mobile WiMAX DL-PUSC configuration for $P_{\mathrm{fa}}=0.02$ and $P_{\mathrm{fa}}=$ 0.001 . We observe that the GLRT still conserves its $1 \mathrm{~dB}$ gain noticed in the previous context. In addition to this performance gain, the GLRT also extracts and provides several additional information, e.g., it estimates the noise variance and the channel response which are very good metrics that can be used to evaluate the link between the cognitive user and the base station.

In the derivation of the proposed detectors, it is assumed that $d_{n, k}$ (2) has a CSCWG distribution with 


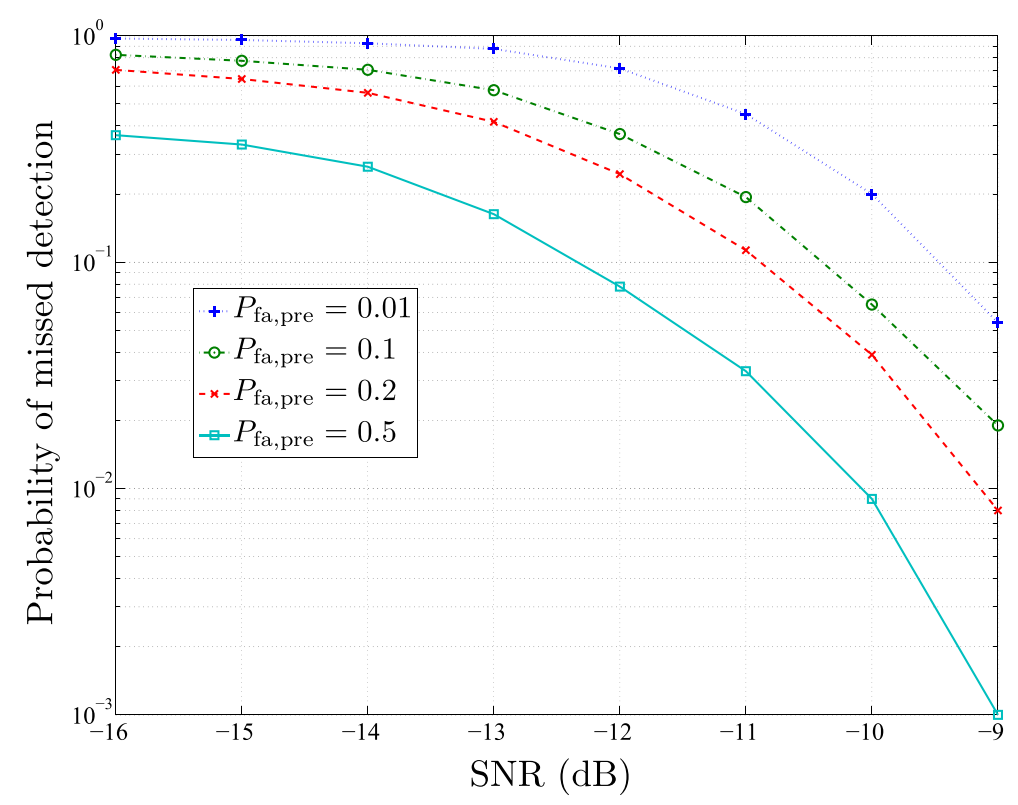

Figure 4 Probability of missed detection of the predetector versus SNR, $N=512, M=24, \mu=0.25 D$.

zero mean and variance of $\sigma_{k}^{2}-\sigma^{2}$. However in practice, this assumption is not necessarily true; and in most standards, this sequence is selected from a set of constellation points and is not a normal random process. To investigate the robustness of the proposed detectors versus this assumption, we have performed a number of simulations where $d_{n, k}$ are randomly and independently chosen from a QPSK, 16-QAM or 64-QAM constellation and compared the results with the case of Gaussian data. For Figure 7 the false alarm rate is set to $P_{\mathrm{fa}}=0.02$ and the sequence $d_{n, k}$ is generated to have either a Gaussian pdf or a nonGaussian pdf. In the non-Gaussian case, $d_{n, k}$ generated as $Q$-QAM sequence where $Q$ is 4,16 , or 64 with equal probabilities. For each of these distributions, we considered two cases (1) an equal power is allocated to all frequency bins ( $d_{n, k}$ is a white noise) and (2) the transmitter allocates

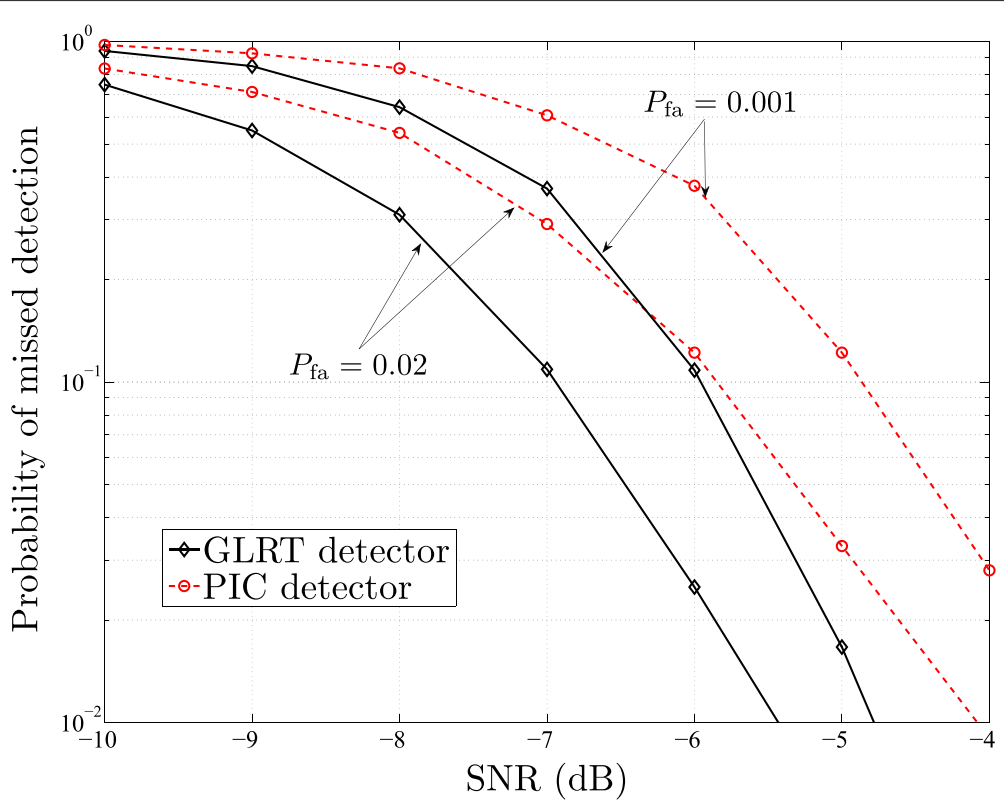

Figure 5 Probability of missed detection of the detector versus SNR, $N=512, M=24, \mu=0.25 D$, under a comb-type configuration of the pilots. 


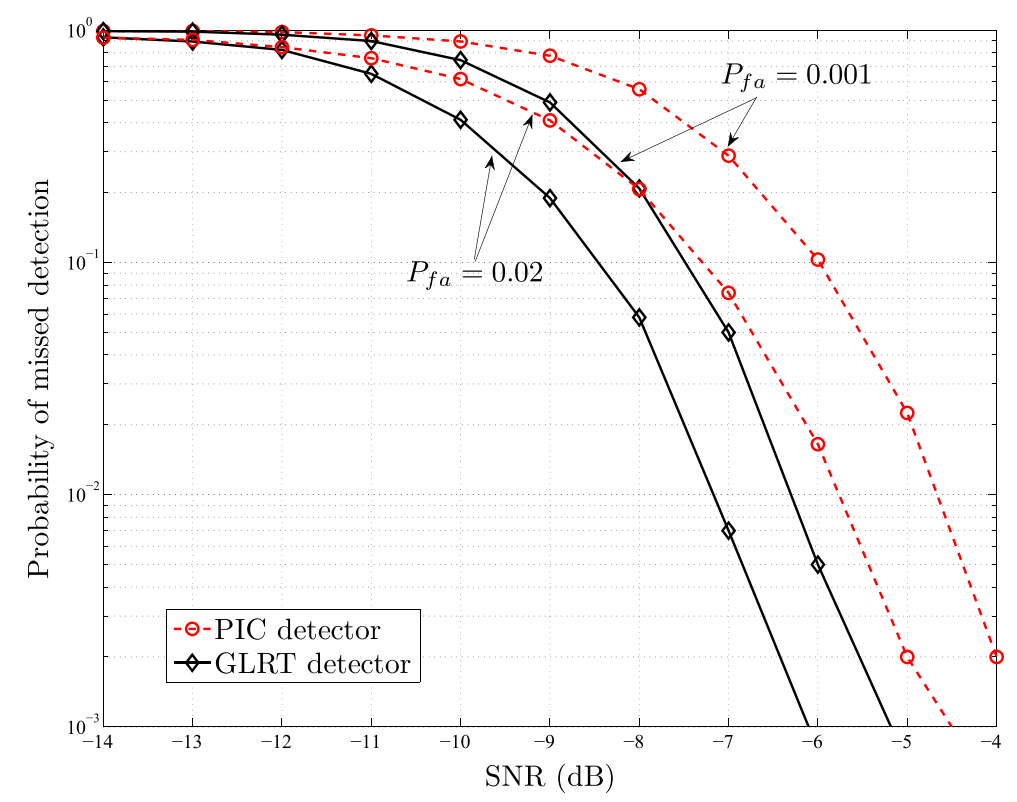

Figure 6 Probability missed detection in the WiMAX DL-PUSC configuration (see Figure 1), $N=512, M=24, \mu=0.25 D$.

different power to different frequency bins (for example, this non-flat power allocation could be the result of a water-filling). For non-uniform power allocation, the power allocated to each frequency bin depends on the channel between the user and the base station which is unknown to the observer. Thus for simplicity, for Q-QAM the allocated power $E\left[\left|d_{n, k}\right|^{2}\right.$ is adjusted/normalized as a function of $Q$ such that the minimum constellation distance is constant for all bins. This figure reveals that the performance proposed detectors are not sensitive the validity of the assumption that $d_{n, k}$ is Gaussian. However, the power allocation has significant impact on the detection performance of all algorithms.

In Figure 8, the receiver operating characteristic (ROC) curves are plotted for both pre-detector and detector and different number of observed symbols $M$. As expected the performance of the algorithm increases as $M$ increase. This is justified intuitively, as more samples

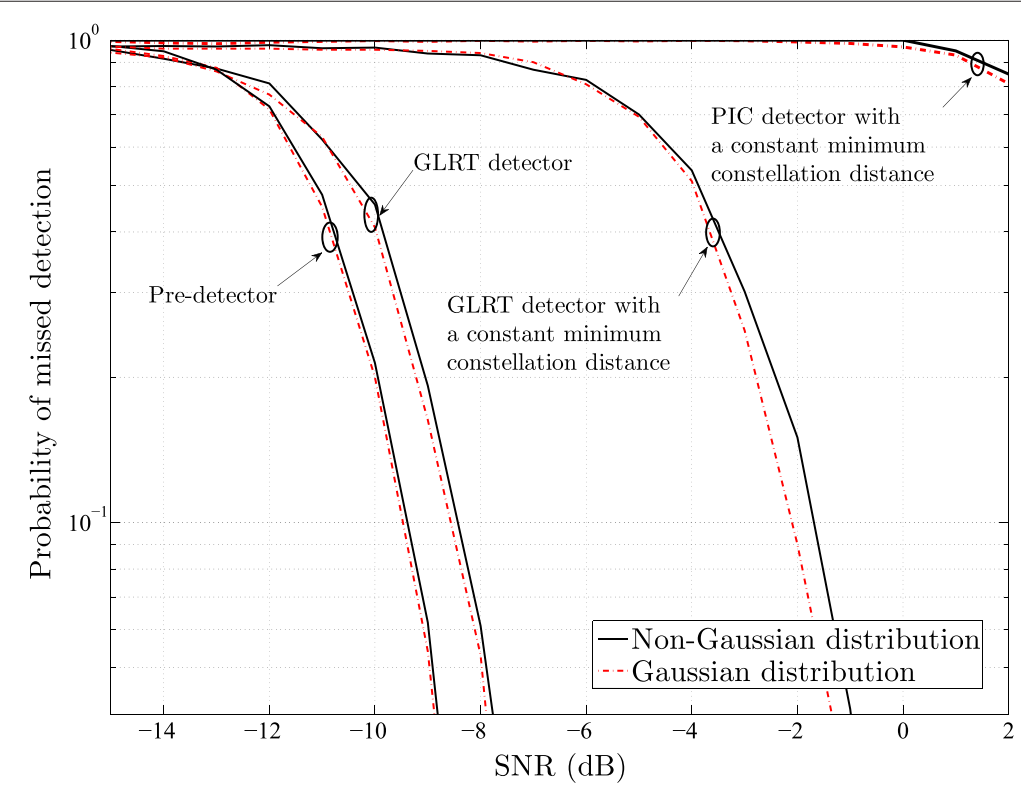

Figure 7 Performance the GLRT detector, the pre-detector and the PICD where $d_{n, k}$ is generated in different ways as either a Gaussian random sequence or as $Q-Q A M$ sequence, where $Q$ randomly is 4,16 , or 64 with compared for $P_{\mathrm{fa}}=0.02, \mu=0.25 D$. 


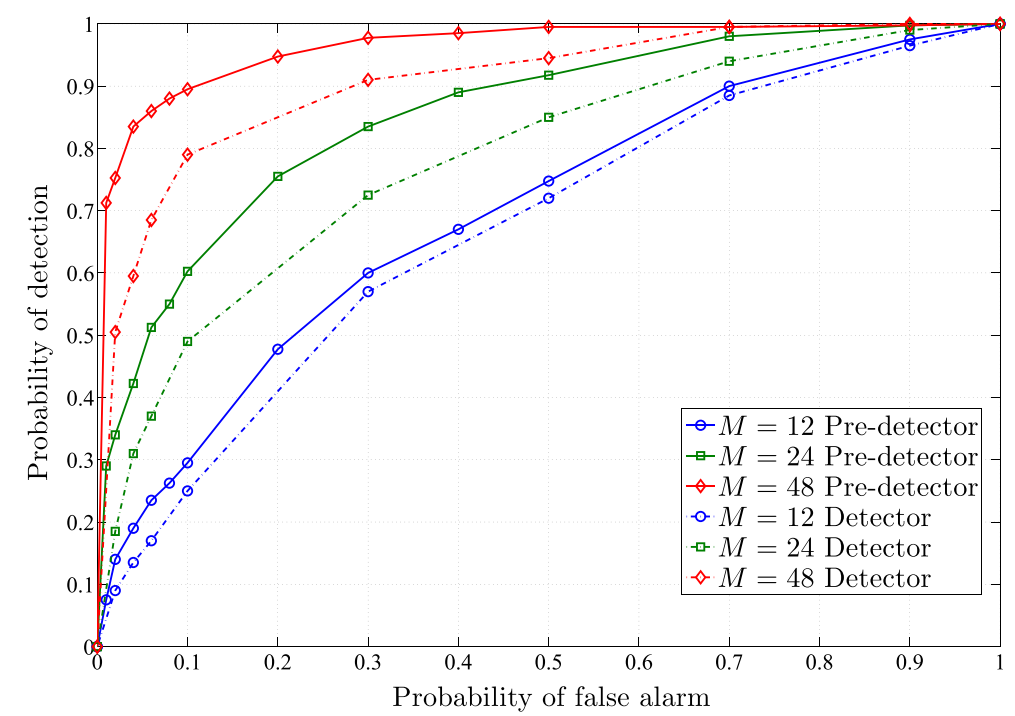

Figure 8 Receiver operating characteristic curves for different number of observed OFDM frames values, $\mathrm{SNR}=-12 \mathrm{~dB}, N=512$, $\mu=0.25 D$, for the WiMAX DL-PUSC configuration (see Figure 1).

results in more information and more accurate estimation of unknown parameters and hence the detection performance improves. Unfortunately we can not increase $M$ arbitrarily since it determines the acquisition time (the time lag before a decision is made), for instance 12, 24 , and 48 observed OFDM symbols represent $1.25,2.5$, and
$5 \mathrm{~ms}$ time-lag for WiMAX Mobile. Thus in practice, these curves represent the trade-off between the time-lag $M$ and $\left(P_{\mathrm{fa}}, P_{\mathrm{fa}}, \mathrm{pre}\right)$ for a given required performance.

In Figure 9, the performance of the cascade combination of the pre-detection and the GLRT is compared with that of the PICD and that of the proposed GLRT

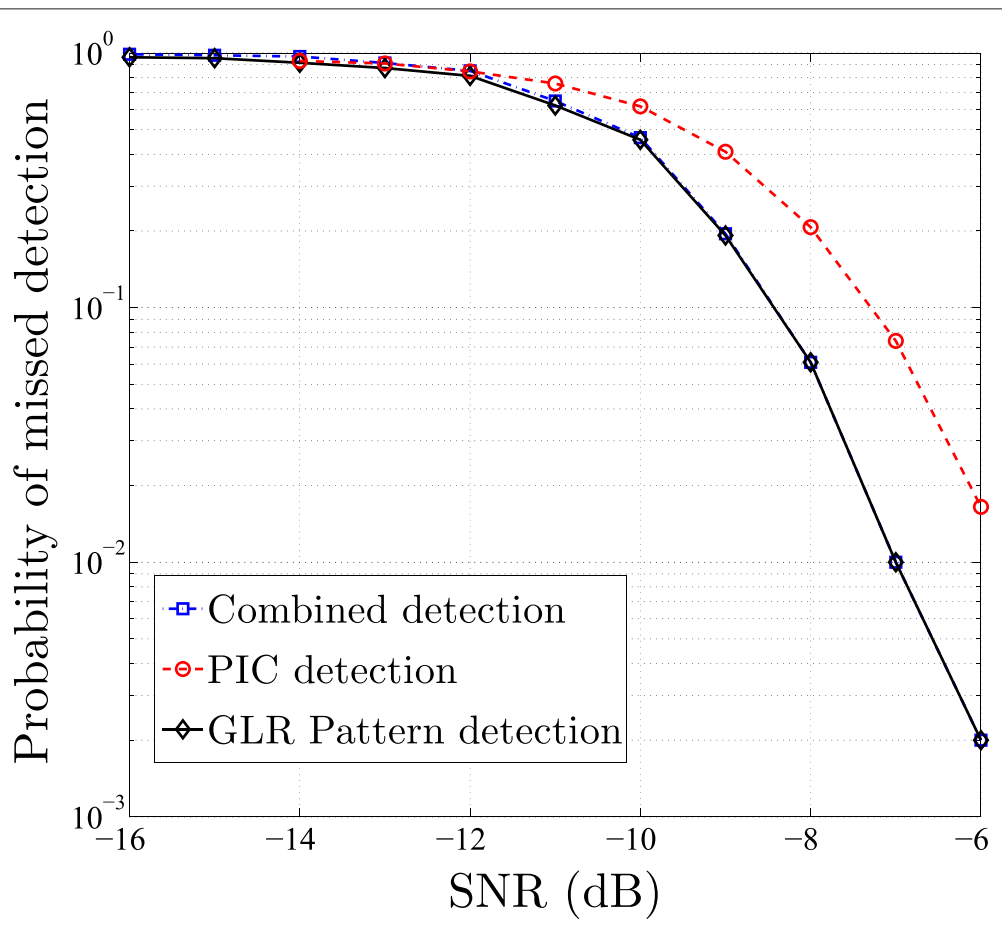

Figure 9 Probability missed detection in the WiMAX DL-PUSC configuration (see Figure 1), for a combined use of pre-detection and detection, $P_{\mathrm{fa}, \mathrm{pre}}=0.2, P_{\mathrm{fa}}=0.02, N=512, M=24, \mu=0.25 D$. 
without any pre-detection. The curves for the GLRT and the PICD are plotted for $P_{\mathrm{fa}}=0.02$. For the cascade combination, first the pre-detection in (24) is performed with a $P_{\mathrm{fa}, \mathrm{pre}}=0.2$. Then if any signal is pre-detected, the identification algorithm in (20) is performed with a $P_{\mathrm{fa}}=0.02$. We observe that their performance are very similar. This is justified by the fact that the pre-detector performs well in the SNR operating range, i.e., the missed detection probability of the pre-detector is very small and it is very unlikely that potential candidate is rejected by the pre-detector. This is why the performance of the cascade combination is not deteriorated compared with that of GLRT.

Figure 10 highlights the effect of time varying channels on the performance of the GLRT detector. Time variation has been simulated using Jake's model [44] for values of the maximum Doppler frequencies $f_{d}=0,100$ and $200 \mathrm{~Hz}$ and a false alarm rate of $P_{\mathrm{fa}}=0.02$. We clearly observe that the proposed method is also robust to Doppler spread. For example for a doppler of $200 \mathrm{~Hz}$, both algorithms lose about $1.33 \mathrm{~dB}$.

\subsection{Asynchronous case}

All derivations in this article so far was based on the assumption that the observer is fully synchronized with the active systems. Unfortunately in practice, the observer could not be synchronized with the systems which are not detected yet. Thus here, we consider the case in which $(\varepsilon, \tau, \varphi)$ defined in (5) is not zero. It is easy to see that the phase shift mismatch $\varphi$ has no impact on the proposed algorithms, since the decision statistics are invariant to the transformation $G(y(m))=y(m) e^{j \varphi}$. However, the frequency offset $\varepsilon$ and the time delay $\tau$ result in inter-carrier interference (ICI) and inter-symbol interference (ISI), respectively. In other words, the impact of $\varepsilon$ and $\tau$ appear as an unknown shift in frequency and time, respectively. A number of techniques are proposed in the literature like Schmidl-Cox [45], Minn-Bhargava [46] or ShiSerpedin [47]. Unfortunately, these techniques require that a periodic pattern to be present in the signal. This requirement is practically not satisfied for the defined standards. Moreover, the cognitive receiver can only process a random portion of the signal; this makes it highly unlikely to pick up one OFDM symbol containing the periodic pattern.

To overcome this problem we propose another approach. After applying the FFT to the data instead of (6), we obtain

$$
Y_{n, k} \triangleq \frac{1}{\sqrt{N}} \sum_{m=0}^{N-1} y[n(N+D)+D+m-\tau] e^{-2 i \pi\left(\frac{k m}{N}+\varepsilon\right)}
$$

For the synchronization purpose, we search on all discrete values of $\tau$ as a multiple integer factor of $N+D$ and $\varepsilon$ as a multiple integer factor of inter carrier frequency spacing. So, we could either shift in time and in frequency the received signal to match the pilot pattern or equivalently shift back the pilot pattern to math the unknown discrete

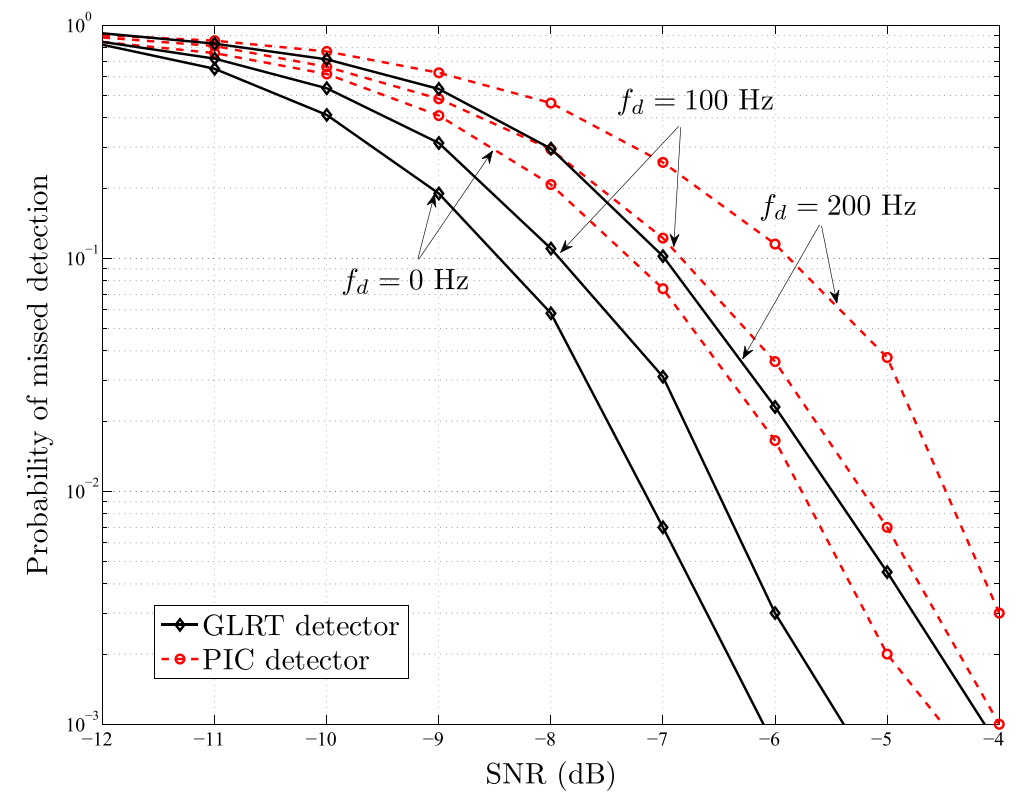

Figure 10 Probability missed detection in the WiMAX DL-PUSC configuration (see Figure 1), for different maximum Doppler frequency values, $P_{\mathrm{fa}}=0.02, N=512, M=24, \mu=0.25 D$. 
values of $(\varepsilon, \tau)$. Let $\mathcal{P}_{\varepsilon, \tau}$ denotes the known pilot pattern shifted back in frequency $\varepsilon$ and in time $\tau$. We define the following decision statistic

$$
\max _{(\varepsilon, \tau)}\left\{\mathcal{T}\left(\mathbf{Y}, \mathcal{P}_{\varepsilon, \tau}\right)\right\} \underset{\mathcal{H}_{0}}{\stackrel{\mathcal{H}_{1}}{\gtrless}} \eta
$$

where $\mathcal{T}$ is defined in (20). It is obvious that the $(\widehat{\varepsilon}, \widehat{\tau})=$ $\arg \max \left\{\mathcal{T}\left(\mathbf{Y}, \mathcal{P}_{\varepsilon, \tau}\right)\right\}$ are maximum likelihood estimates of $\varepsilon, \tau$ $\varepsilon$ and $\tau$. Thus, this detector jointly performs synchronization along with the detection. This is highlighted in Figure 11, where the values of the test statistic are plotted for different frequencies and timing offsets using a signal model similar to the one depicted in Figure 1 for a fully synchronized case. The SNR is set to $0 \mathrm{~dB}$, the number of observed OFDM symbols is set to $M=24$. The time offset is varying in the interval $\tau \in[-0.5(N+D), 0.5(N+D)]$ and the frequency offset $\varepsilon \in[-0.5,0.5]$. The Figure shows that the test statistic is maximum for $\varepsilon=0$ (no ICI) and $\tau=0$ (no ISI) which confirms that $\widehat{\varepsilon}, \widehat{\tau}$ are the ML estimates of $\varepsilon$ and $\tau$.

To study the impact of this approach, we performed simulations where the received signal is suffering from a discrete time-frequency mismatch such that $\tau \in[-0.5(N+$ $D), 0.5(N+D)]$ and the frequency offset $\varepsilon \in[-0.5,0.5]$. The maximization described in 29 is achieved by exploring discrete value within the two described intervals. Each interval is sampled into 11 uniformly distributed values. Thus we have to compute 121 values of the decision statistic, the maximum is achieved when the couple $(\hat{\tau}, \hat{\varepsilon})$ is nearest to the true values $(\tau, \varepsilon)$. In order to keep the false alarm rate to the expected value, maximizing the cost function implies to adjust the detection threshold. In fact, if $K_{\varepsilon}$ and $K_{\tau}$ denote the number of discrete frequency and time shifts, respectively, then $K_{\varepsilon} K_{\tau}$ test statistics have to be computed. Assuming that under $\mathcal{H}_{0}$ the test statistic values are independent from one shift to another. Thus, the detection threshold has to verify

$$
\left(\mathcal{F}_{\mathcal{T}, \mathcal{H}_{0}}\left(\eta_{\text {det }}\right)\right)^{K_{\varepsilon} K_{\tau}}=1-P_{\text {fa }} .
$$

The obtained results for a WiMAX configuration are plotted in Figure 12. Compared to the synchronous case (Figure 6), we first remark that both algorithms suffer a performance loss of about $1 \mathrm{~dB}$. In addition to this common loss, the proposed GLRT is also loosing about $1 \mathrm{~dB}$ gain against the PIC detector. This is due to the shape of the test statistic (Figure 11 ) versus $(\varepsilon, \tau)$. This shows that for the synchronization our criterion compared to the PIC criterion is much sharper and has faster decay around the true value $(\varepsilon, \tau)$.

The computational complexity of (29) is $K_{\varepsilon} K_{\tau}$ times that of (20). However, employing the cascade combination and using a judiciously chosen value $P_{\mathrm{fa}}$,pre, the average computational cost is considerably reduced. In this case, if no signal is present less computational resource is wasted for synchronization. Under $\mathcal{H}_{0}$, the computational cost is $\mathrm{CC}_{0}=\mathrm{CC}_{\text {pre }}+K_{\varepsilon} K_{\tau} P_{\mathrm{fa} \text {,pre }} \mathrm{CC}_{\mathrm{GLRT}}$, where $\mathrm{CC}_{\text {pre }}$ and $\mathrm{CC}_{\mathrm{GLRT}}$ are the computational cost of the pre-detector and the GLRT detector, respectively. Under $\mathcal{H}_{1}$, the computational cost is $\mathrm{CC}_{1}=\mathrm{CC}_{\text {pre }}+K_{\varepsilon} K_{\tau} P_{\mathrm{d}, p r e} \mathrm{CC}_{\mathrm{GLRT}}$, where $P_{\mathrm{d}, p r e}$ is the detection probability of the pre-

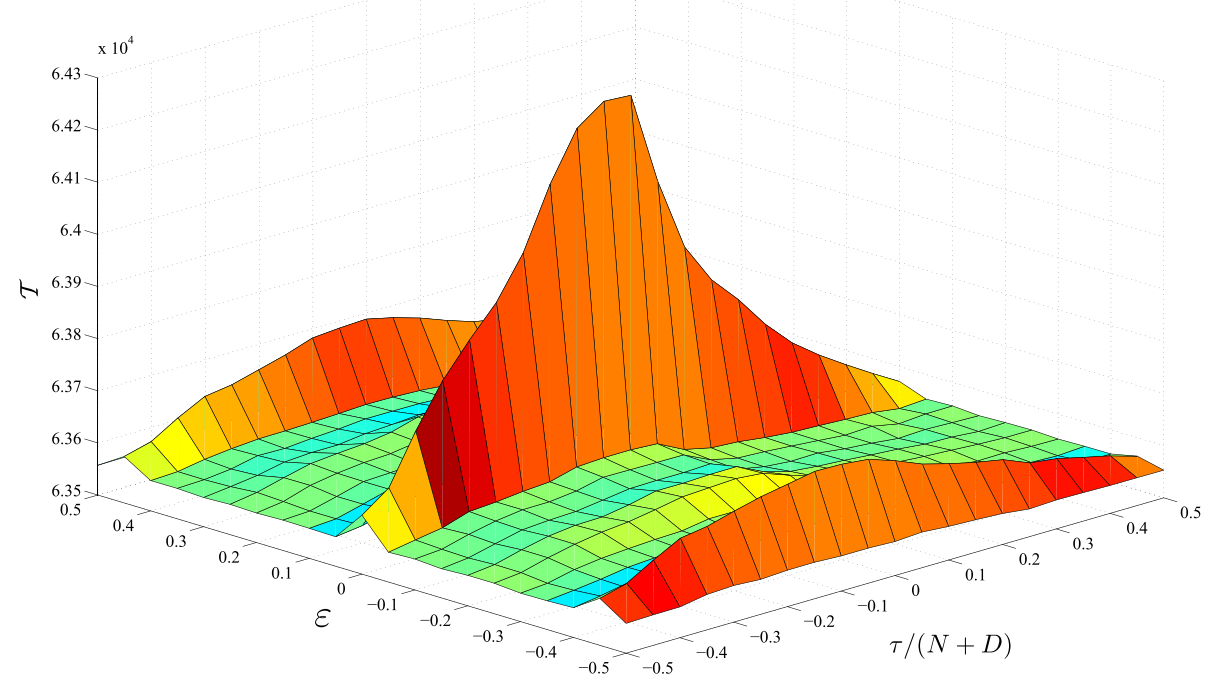

Figure 11 Effect of synchronization impairment on test statistic value, SNR $=0 \mathrm{~dB}$, WiMAX DL-PUSC configuration (see Figure 1), $N=512, M=24, \mu=0.25 D, P_{\mathrm{fa}}=0.002$. 


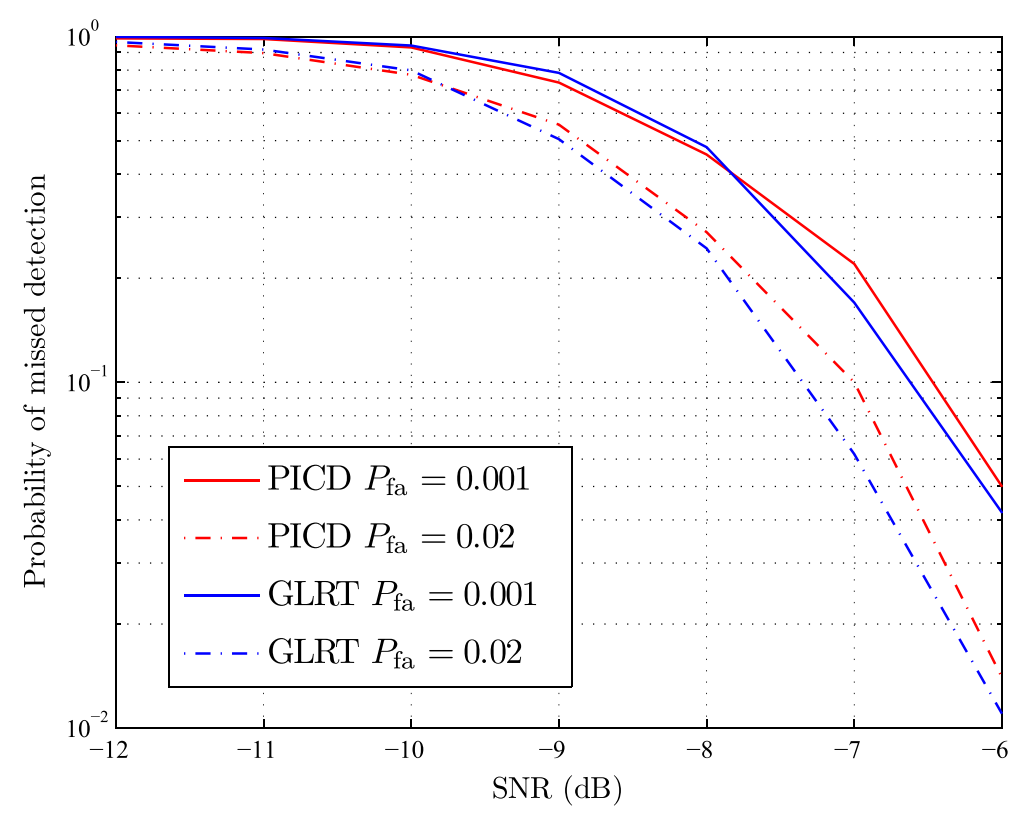

Figure 12 Effect of synchronization impairment on the performance of the proposed algorithm, WiMAX DL-PUSC configuration (see Figure 1), $N=512, M=24, \mu=0.25 D, \tau \in[-0.5(N+D), 0.5(N+D)]$ and $\varepsilon \in[-0.5,0.5]$.

detector. The overall $\mathrm{CC}$ of the receiver is $\mathrm{CC}=$ $P\left[\mathcal{H}_{0}\right] C_{0}+\left(1-P\left[\mathcal{H}_{0}\right]\right) C C_{1}$. Figures 13 , illustrate the overall computational cost of the receiver versus $P_{\mathrm{fa}}$,pre for different values of $P_{0}$ and SNR. The CC increases as $P_{\mathrm{fa}}$,pre increases, and thus, a judicious choice on $P_{\text {fa,pre }}$ has to be done to maintain a good performance of the pre-detector providing a reasonable overall CC.

\subsection{Discussion on the case where the data symbols are PSK signals}

For current and most known future systems, the pilot symbols are PSK signals. This particularity has been exploited in this article and allows us to make use of the algorithm proposed in [34]. For high SNRs between the BS and the receiver, the data symbols are drawn

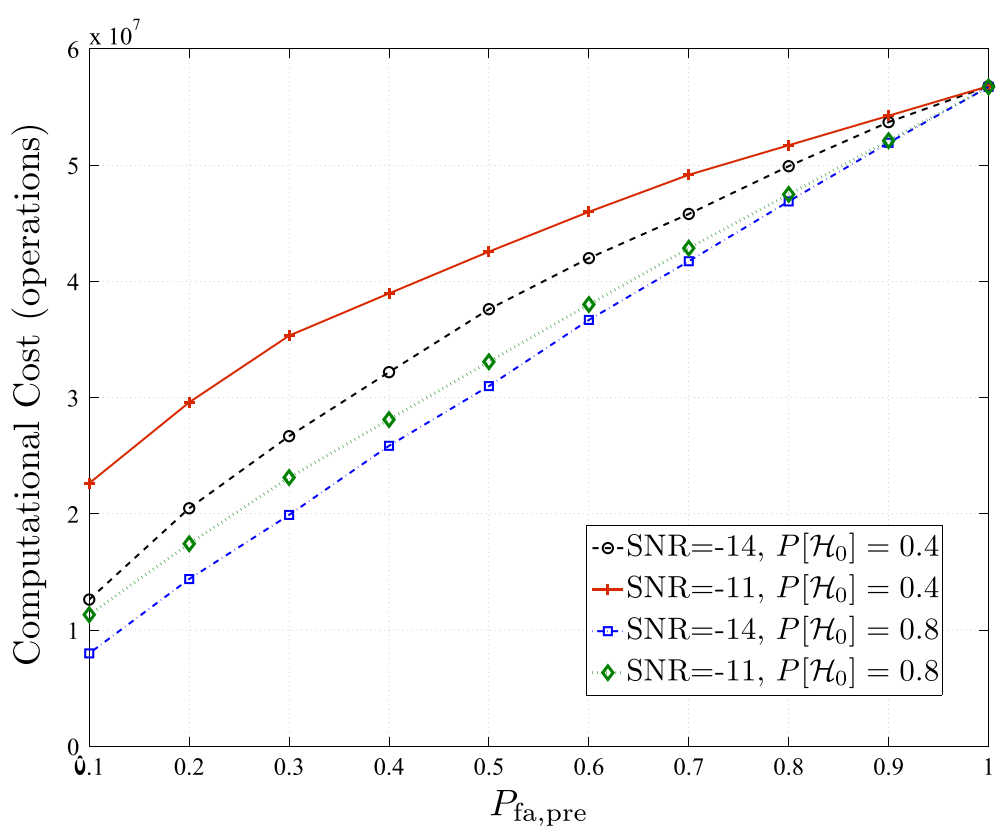

Figure 13 Computational cost of the receiver versus SNR for various values of $P_{0}$. 


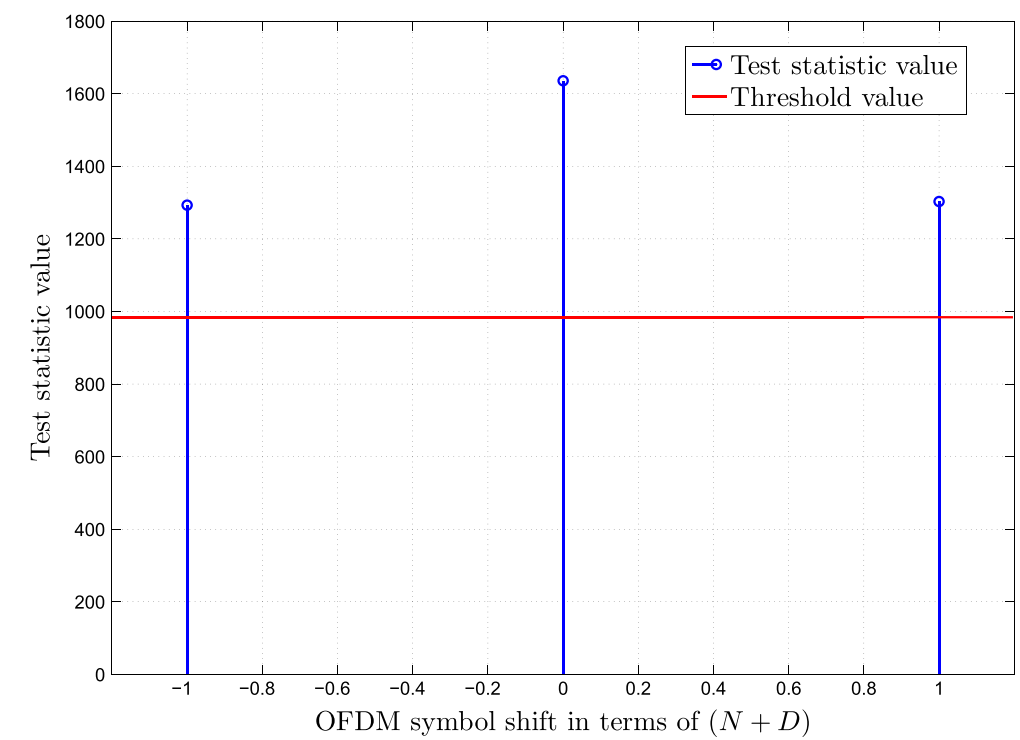

Figure 14 Behavior of the test statistic in a case where BPSK symbols are present everywhere, the pilots are BPSK symbols $2.54 \mathrm{~dB}$ boosted, SNR $=0 \mathrm{~dB}$, WiMAX DL-PUSC configuration (see Figure 1), $N=512, M=24, \mu=0.25 D$.

from a large number of constellation points such as 16QAM or 64-QAM. However as an observer, the cognitive device can intercept a communication between a BS and a mobile with a low SNRs, where to fit the BER requirement the number of constellation points is reduced. Thus, we observe BPSK or QPSK signals not only on pilot positions but also on the data positions in the sub-carriers with lower SNRs. Since our method relies on the pattern of PSK signals, a false alarm can be triggered when the pilot pattern is absent but when the tested position contains PSK signals of the same order of the desired pilot pattern, misleading our decision. However, we can get around this problem exploiting the fact that the pilots are always boosted in power in the existing standards (for example, $3 \mathrm{~dB}$ for WiFi, $2.54 \mathrm{~dB}$ for WiMax). This power difference could be easily exploited to identify the pilot pattern using an exhaustive search similar to (29). Figure 14 shows the test statistic value for the signal model plotted in Figure 1. The length of the pilot pattern is equal to 2, thus we computed $\mathcal{T}$ when shifting the signal by one OFDM symbol forward and backward. As expected, the test statistic values are greater than the threshold, however, the test statistic is maximum only for the correct position of the pattern since the pilots are boosted.

\section{Conclusion}

In this article, we presented a new method for the detection of active OFDM based systems. The proposed method exploit the pilot pattern embedded into existing standards as a characteristic for such a detection and is based on a GLR test. The presented algorithm is computationally inexpensive and does not require any overhead or modification into the actual structure of the PHY layer of the networks of interest. The performance of the proposed method is compared against the PICD proposed in [24]. The proposed technique outperforms the pilot based identifiers proposed in the literature and shows an equivalent robustness to the propagation environment. Since this algorithm, in contrast to the PICD, does not exploit any a-priori information about the pilot correlation it could be employed for all other existing standards such as LTE, DVB-T. We also proposed another GLRTbased pre-detector which ignores the pilot information and is just used as a filter in order to reduce the computational cost for the cases where a large number of possible systems/patterns need to be tested. Moreover as a side product, the proposed detector performs noise variance estimation, channel magnitude estimation and time-frequency synchronization. These extracted information can be used to sense the quality of service of the detected network.This knowledge will also help the user to chose the network offering the best link quality, approaching the always best connected concept.

\section{Endnote}

${ }^{a}$ e.g., when observing 24 symbols $(2.5 \mathrm{~ms})$ in the WiMAX standards using 512 sub-carriers $M N$ costs 12288.

${ }^{\mathrm{b}}$ Note that as we can see in Equation (20), the test statistic does not depend on the pilots power. Thus, the algorithm works even if the pilot and data tones have the same power.

\section{Competing interests}

The authors declare that they have no competing interests. 


\section{Author details}

${ }^{1}$ Institut Telecom, Telecom Bretagne, UMR CNRS 3192 Lab-STICC, Université Européenne de Bretagne, Bretagne, France. ${ }^{2}$ Electrical and Computer Engineering Department, Queen's University, Kingston, Ontario, Canada.

Received: 17 July 2012 Accepted: 7 February 2013

Published: 15 March 2013

\section{References}

1. U.S. FCC, Review of Spectrum Management Practicies. Tech. Rep. Federal Communications Commission (2002)

2. U.S. FCC, Second Report and Order and Memorandum Opinion and Order, in the Matter of Unlicensed Operation in the TV Broadcast Bands Additional Spectrum for Unlicensed Devices Below $900 \mathrm{MHz}$ and in the $3 \mathrm{GHz}$ Band. Tech. Rep. Federal Communications Commission (2008)

3. IEEE 802.22 WG, IEEE 802.22 Working Group on Wireless Regional Area Networks Enabling Rural Broadband Wireless Access Using Cognitive Radio Technology. http://www.ieee802.org/22/. [Online; accessed 24-Sep-2010]

4. J Mitola, GQ Maguire, Cognitive radio: making software radios more personal. IEEE Personal Commun. 6(4), 13-18 (1999)

5. S Haykin, Cognitive radio: brain-empowered wireless communications IEEE J. Sel. Areas Commun. 23(2), 201-220 (2005)

6. U.S. FCC, Notice of Proposed Rulemaking, in the Matter of Unlicensed Operation in the TV Broadcast Bands (ET Docket no. 04-186) and Additional Spectrum for Unlicensed Devices below $900 \mathrm{MHz}$ and in the 3 GHz Band. Tech. Rep. FCC ET Docket 04-113 (2004)

7. E Gustafsson, A Jonsson, Always best connected. IEEE Trans. Wirel. Commun. 10(1), 49-55 (2003)

8. Z Dai, R Fracchia, J Gosteau, P Pellati, G Vivier, in IEEE International Conference on Communications, ICC'08. Vertical Handover Criteria and Algorithm in IEEE802.11 and 802.16 Hybrid Networks, (2008), pp. $2480-2484$

9. J McNair, F Zhu, Vertical handoffs in fourth-generation multinetwork environments. IEEE Trans. Wirel. Commun. 11(3), 8-15 (2004)

10. H Urkowitz, Energy detection of unknown deterministic signals. Proc. IEEE. 55(4), 523-531 (1967)

11. D Cabric, SM Mishra, RW Brodersen, in Conference on Signals, Systems and Computers, 2004. Conference Record of the Thirty-Eighth Asilomar, vol. 1. Implementation issues in spectrum sensing for cognitive radios (IEEE, pp. $772-776$

12. C Stevenson, G Chouinard, Z Lei, W Hu, S Shellhammer, W Caldwell, IEEE 802.22: the first cognitive radio wireless regional area network standard. IEEE Commun. Mag. 47(1), 130-138 (2009)

13. D Birru, S Shankar, C Cordeiro, K Challapali, IEEE 802.22: an introduction to the first wireless standard based on cognitive radios. J. Commun. 1(1), 38-47 (2006)

14. A Bouzegzi, P Ciblat, Jallon, New algorithms for blind recognition of OFDM based systems. Elsevier Signal Process. 90(3), 900-913 ((2010))

15. A Punchihewa, VK Bhargava, C Despins, Blind estimation of OFDM parameters in cognitive radio networks. IEEE Trans. Wirel. Commun. 10(3), 733-738 (2011)

16. N Han, G Zheng, SH Sohn, JM Kim, in 4th International Conference on Wireless Communications, Networking and Mobile Computing, WiCOM'08. Cyclic autocorrelation based blind OFDM detection and identification for cognitive radio, (2008), pp. 1-5

17. M Oner, $F$ Jondral, On the extraction of the channel allocation information in spectrum pooling systems. IEEE J. Sel. Areas Commun. 25(3), 558-565 (2007)

18. T Yucek, H Arslan, OFDM signal identification and transmission parameter estimation for cognitive radio applications, (2007), pp. 4056-4060

19. H Li, Y Bar-Ness, A Abdi, OS Somekh, W Su, in 1st International Conference on Cognitive Radio Oriented Wireless Networks and Communications. OFDM modulation classification and parameters extraction, (2006), pp. 1-6

20. A Al-Habashna, OA Dobre, R Venkatesan, DC Popescu, in IEEE International Conference on Communications (ICC). Joint cyclostationarity-based detection and classification of mobile wimax and Ite ofdm signals, (2011), pp. 1-6
21. PD Sutton, KE Nolan, LE Doyle, Cyclostationary signatures in practical cognitive radio applications. IEEE J. Sel. Areas Commun. 26(1), 13-24 (2008)

22. K Maeda, A Benjebbour, T Asai, T Furuno, T Ohya, in IEEE 2nd International Symposium on New Frontiers in Dynamic Spectrum Access Networks, DYSPAN. Recognition among OFDM-based systems utilizing cyclostationarity-inducing transmission, (2007), pp. 516-523

23. K Maeda, A Benjebbour, T Asai, T Furuno, T Ohya, in Eurasip Journal on Wireless Communications and Networking. Cyclostationarity-inducing transmission methods for recognition among OFDM-based systems, (2008), pp. 1-14, 10.1155/2008/586172

24. F-X Socheleau, S Houcke, P Ciblat, A Aissa-El-Bey, Cognitive OFDM system detection using pilot tones second and third-order cyclostationarity. Elsevier Signal Process. 91(2), 1-17 (2011)

25. Y-H Jung, YH Lee, Use of periodic pilot tones for identifying base stations of FH-OFDMA systems. IEEE Commun. Lett. 10(3), 192-194 (2006)

26. A Al-Habashna, OA Dobre, R Venkatesan, Popescu D C, in GLOBECOM 2010, 2010 IEEE Global Telecommunications Conference. Cyclostationarity-based detection of Ite ofdm signals for cognitive radio systems, (2010), pp. 1-6

27. AJ Coulson, Maximum likelihood synchronization for OFDM using a pilot symbol: algorithms. IEEE J. Sel. Areas Commun. 19(12), 2486-2494 ((2001))

28. TM Schmidl, DC Cox, Robust frequency and timing synchronization for OFDM. IEEE Trans. Commun. 45(12), 1613-1621 (1997)

29. A Viholainen, T Stitz, T Ihalainen, M Renfors, in EURASIP Journal on Advances in Signal Processing, vol. 2010:741429. Pilot-based synchronization and equalization in filter bank multicarrier communications (EURASIP, 2010), pp. 1-18

30. Y Li, Pilot-symbol-aided channel estimation for OFDM in wireless systems. IEEE Trans. Veh. Technol. 49(4), 1207-1215 (2000)

31. S Colieri, M Ergen, A Puri, A Bahai, in IEEE 56th Vehicular Technology Conference, 2002. Proceedings. VTC 2002-Fall, vol. 2. A study of channel estimation in OFDM systems (IEEE, 2002), pp. 894-898

32. S Colieri, M Ergen, A Puri, A Bahai, Channel estimation techniques based on pilot arrangement in OFDM systems. IEEE Trans. Broadcast. 48(3), 223-229 (2002)

33. IEEE Std 802.16, Part 16: air interface for broadband wireless access systems, Amendment 2: Physical and Medium Access Control layers for Combined Fixed and Mobile Operation in License Bands and Corrigendum, IEEE, vol. 1(2005), pp. 1-822

34. S Gazor, M Derakhtian, AA Tadaion, Computationally efficient maximum likelihood sequence estimation and activity detection for M-PSK signals in unknown flat fading channels. IEEE Signal Process. Lett. 17(10), 871-874 (2010)

35. AA Tadaion, M Derakhtian, S Gazor, MM Nayebi, MR Aref, Signal activity detection of phase-shift keying signals. IEEE Trans. Commun. 54(6), 1143-1143 (2006)

36. E Axell, EG Larsson, Optimal and sub-optimal spectrum sensing of OFDM signals in known and unknown noise variance. IEEE J. Sel. Areas Commun. 29(2), 290-304 (2011)

37. S Bokharaiee, HH Nguyen, E Shwedyk, Blind spectrum sensing for OFDM-based cognitive radio systems. IEEE Trans. Veh. Technol. 60(3), 858-871 (2011)

38. H shin Chen, W Gao, D Daut, Spectrum sensing for OFDM systems employing pilot tones. IEEE Trans. Wirel. Commun. 8(12), 5862-5870 (2009)

39. J Font-Segura, $X$ Wang, Glrt-based spectrum sensing for cognitive radio with prior information. IEEE Trans. Commun. 58(7), 2137-2146 (2010)

40. RE Glaser, The ratio of the geometric mean to the arithmetic mean for a random sample from a gamma distribution. J. Am. Stat. Assoc. 71(354), 480-487 (1976)

41. S-M Kay, Fundamentals of Statistical Signal Processing, Volume Il: Detection Theory, ((Prentice Hall, Upper Saddle River, 1998). ISBN 0-13-504135-X

42. IF Akyildiz, W-Y Lee, MC Vuran, S Mohanty, Next generation/dynamic spectrum access/cognitive radio wireless networks: a survey. Comput. Netw. Elsevier. 50(13), 2127-2159 (2006)

43. F-K Jondral, Software-defined radio basics and evolution to cognitive radio. EURASIP J. Wirel.Commun. Network. 3, 275-283 (2005)

44. WC Jakes, Microwave Mobile Communications. (John Wiley and Sons Inc., New York, 1975)

45. CWilliams, MA Beach, S McLaughlin, Robust OFDM timing synchronisation. Electron. Lett. 41(13), 751-752 (2005) 
46. H Minn, VK Bhargava, in 2000 IEEE 51st Vehicular Technology Conference Proceedings, vol. 1. A simple and efficient timing offset estimation for OFDM systems, VTC 2000-Spring Tokyo, 2000), pp. 51-55

47. K Shi, E Serpedin, Coarse frame and carrier synchronization of ofdm systems: a new metric and comparison. IEEE Trans. Wirel. Commun. 3(4), 1271-1284 (2004)

doi:10.1186/1687-1499-2013-72

Cite this article as: Oularbi et al.: GLR test for OFDM system identification using pilot tones pattern. EURASIP Journal on Wireless Communications and Networking 2013 2013:72.

Submit your manuscript to a SpringerOpen ${ }^{\circ}$ journal and benefit from:

- Convenient online submission

- Rigorous peer review

- Immediate publication on acceptance

- Open access: articles freely available online

- High visibility within the field

- Retaining the copyright to your article

Submit your next manuscript at $\gg$ springeropen.com 